\title{
Energy Efficiency of Ultra-Low-Power Bicycle Wireless Sensor Networks Based on a Combination of Power Reduction Techniques
}

\author{
Sadik Kamel Gharghan, ${ }^{1,2}$ Rosdiadee Nordin, ${ }^{1}$ and Mahamod Ismail ${ }^{1}$ \\ ${ }^{1}$ Department of Electrical, Electronic and Systems Engineering, Faculty of Engineering and Built Environment, \\ Universiti Kebangsaan Malaysia (UKM), 43600 Bangi, Selangor, Malaysia \\ ${ }^{2}$ College of Electrical and Electronic Engineering Techniques, Middle Technical University, Baghdad, Iraq
}

Correspondence should be addressed to Sadik Kamel Gharghan; sadiq_gharghan@yahoo.com

Received 12 April 2016; Accepted 17 July 2016

Academic Editor: Eduard Llobet

Copyright (C) 2016 Sadik Kamel Gharghan et al. This is an open access article distributed under the Creative Commons Attribution License, which permits unrestricted use, distribution, and reproduction in any medium, provided the original work is properly cited.

In most wireless sensor network (WSN) applications, the sensor nodes (SNs) are battery powered and the amount of energy consumed by the nodes in the network determines the network lifespan. For future Internet of Things (IoT) applications, reducing energy consumption of SNs has become mandatory. In this paper, an ultra-low-power nRF24L01 wireless protocol is considered for a bicycle WSN. The power consumption of the mobile node on the cycle track was modified by combining adjustable data rate, sleep/wake, and transmission power control (TPC) based on two algorithms. The first algorithm was a TPC-based distance estimation, which adopted a novel hybrid particle swarm optimization-artificial neural network (PSO-ANN) using the received signal strength indicator (RSSI), while the second algorithm was a novel TPC-based accelerometer using inclination angle of the bicycle on the cycle track. Based on the second algorithm, the power consumption of the mobile and master nodes can be improved compared with the first algorithm and constant transmitted power level. In addition, an analytical model is derived to correlate the power consumption and data rate of the mobile node. The results indicate that the power savings based on the two algorithms outperformed the conventional operation (i.e., without power reduction algorithm) by $78 \%$.

\section{Introduction}

Wireless sensor networks (WSNs) consisting of several wireless nodes deployed in a vast area have the capacity to sense different events or parameters according to the type of application. The sensed parameters are determined using sensor nodes (SNs) and transmitted directly or through the router node to the coordinator node. The SNs, called motes in the WSN applications, are typically characterized by low power consumption, miniaturized size, and low cost [1]. In WSNs, SNs are randomly deployed to collect data from an interested area $[2,3]$. The radio frequency unit of these nodes establishes communication between the nodes of the network and communication of the network with the outside world. The radio frequency and processing units consume the most energy [4]. Therefore, reducing the power consumption in the communication unit for these nodes is considered a major challenge in WSNs [5]. In some cases, a transmission of one byte consumes more power than the power required to execute a thousand instructions [6,7].

The SNs have restricted resources, namely, energy, memory size, communication range, and computational capacity. The SNs require a supply of energy from batteries as a substitute energy source. However, battery power is limited. Therefore, reducing power consumption and extending battery life are compulsory because SNs use battery power as a primary energy source. The SNs become inefficient and lose their sensing and communication capacities when their battery energy is exhausted. Consequently, several strategies and techniques have been used to save energy in WSNs. Several studies have proposed solutions to the energy consumption and battery lifetime problems of SNs in WSNs. These strategies, 
such as reductions in data communication, sleep/wake (S/W) scheduling [8-10], data aggregation [11], selection of the optimal location of the sink node [12], distribution of workload between the sensors in the network [13], data-driven techniques that reduce the amount of sample data, mobility [14], data compression [15], topology control [16], and transmission power control (TPC), have been adopted in different approaches.

WSNs are important in sports applications. In particular, WSNs are used in track cycling sports to monitor athlete performance. However, bicycle WSNs are different from other types of WSNs. For example, (i) radio connectivity is highly sensitive to bike position on the track because of the differences in distance between the cyclist and the coach. Thus, TPCs must adjust the transmission power level according to the bicycle position on the track. (ii) The energy resources are limited (i.e., they must be small to reduce the size and weight of the bicycle). Hence, the nRF24L01 can be used because it has a small size, namely, a size of $29 \times 15 \mathrm{~mm}^{2}$, and is lightweight, at $2.0 \mathrm{~g}$ [17]. The ANT wireless protocol is used to monitor the biomechanical and physiological parameters of the bicycle and cyclist, respectively. (iii) All SNs are mobile on the bike and are battery-constrained devices. Replacing or recharging batteries when the bike is on the track is impossible; thus, energy management is essential. (iv) Memory size is limited to the smallest size of the sensor node. In this case, a load division technique through a master node can be used to avoid intensive computation in the SNs. Finally, (v) harvesting energy as an alternative to the power source cannot be used due to either added extra weight or aerodynamic or rolling resistance, which reduces bike speed and induces fatigue in athletes during cycling. Both aspects are critical in a competitive event.

Several factors, such as (i) transmitted power, (ii) duty cycling (the sensor nodes alternate between active and sleep periods during their lifespan to save energy. It can be achieved through sleep/wake strategy) [18], (iii) data rate, (iv) modulation technique, (v) adaptive sampling, (vi) data aggregation, (vii) data compression, (viii) network topology, and (ix) cooperative communication, directly affect the power consumption of WSNs. In our proposed application, the bicycle moves on the track cycle and carries several SNs. The SN's battery cannot be replaced or charged. Consequently, the power consumption of these mobile nodes can be reduced by combining the first three techniques (i.e., transmitted power, duty cycling, and data rate), which have not been considered together in previous research. The mobile node mounted on a bike is moved to predefined locations (not random) on the cycle track. Therefore, the estimated distance between the bike mobile node and the coach (the master node is located at the center of the track field) can be obtained based on a proposed hybrid particle swarm optimizationartificial neural network (PSO-ANN) algorithm. The ANN algorithm uses the received signal strength indicator (RSSI) values (received by the mobile node) in the training and testing process. The RSSI values can be obtained from real measurement or generated from path loss models. In our application, these values are generated from a log normalshadowing model (LNSM).
The power consumption of the mobile node is reduced by adopting two algorithms. The first algorithm is the TPC-based distance estimation, which primarily depends on distance estimation and provides an accurate distance based on the PSO-ANN algorithm. The mobile node transmitted power and data rate can be adjusted according to the distance between the master and mobile nodes when the bike moves on the track. The second algorithm, which uses the TPCbased accelerometer, represents a novel method of adaptive transmitted power and data rate for mobile nodes. The transmitted power and data rate can be controlled based on the ADXL 335 accelerometer sensor, in which the transmitted power level and data rate can be determined based on the inclination angle (output of accelerometer) of the bicycle as it moves on the track. In the two aforementioned algorithms, three power reduction techniques can be considered. These techniques are (i) the TPC technique, (ii) the S/W strategy, and (iii) the adjustment of the data rate of the ANT. In addition, the TPC technique can be combined with the S/W strategy (TPCS/W) to further reduce the power consumption. The contributions of this paper are as follows:

(i) The power consumption of the master and mobile nodes is modeled with respect to the ANT data rate.

(ii) The novel PSO-ANN algorithm can accurately estimate the distance between the mobile node and the master node.

(iii) The power savings of the mobile node for both outdoor and indoor velodromes are improved based on a proposed TPC-based distance estimation algorithm relative to constant transmitted power.

(iv) Finally, a novel method, namely, one that uses the TPC-based accelerometer algorithm, can significantly reduce the power consumption of the mobile node and all of the ANT modules of the adopted WSN.

\section{Related Research}

Among the many solutions to reducing power consumption in WSNs is the TPC technique. The TPC has already been highlighted in previous research. One study [19] investigated the effect of the TPC using separated power levels on both network lifespan and bandwidth requirements. The authors developed mathematical programming models to identify the effects of transmission power levels and to compare the performances of different discrete transmission power strategies. The network lifetime was extended to $20 \%$ in comparison to the use of a single transmission power. In their study [20], the authors proposed a power control technique in the medium access control (MAC) layer of the ZigBee wireless protocol. The optimally transmitted power was calculated according to the distance between nodes. Bhuvaneswari et al. [2] proposed an anchor node transmitted power control under SN localization. The localization was based on the RSSI of the ZigBee wireless protocol.

Ramakrishnan and Krishna [21] examined the adaptive transmission power of SNs based on fuzzy logic control (FLC) 
TABLE 1: Comparison of power savings in previous works.

\begin{tabular}{|c|c|c|c|c|}
\hline Reference & Wireless technology & Type of study & Metric & Power savings (\%) \\
\hline Cotuk et al. [19] & ZigBee & Experimental & TPC & 20 \\
\hline Ramakrishnan and Krishna [21] & N/A & Simulation & TPC & $40.62(\mathrm{OL}-\mathrm{FTPC})^{*}$ and $38.6(\mathrm{CL}-\mathrm{FTPC})$ \\
\hline Castagnetti et al. [23] & N/A & Simulation & TPC combined with sleep/wake & 26 \\
\hline Kim et al. [25] & ZigBee & Experimental & TPC & 51.63 \\
\hline Ikram et al. [26] & ZigBee & Experimental & TPC combined with AMC! & 50 \\
\hline Correia et al. [27] & ZigBee & Experimental & TPC & 57 \\
\hline
\end{tabular}

${ }^{*}$ Open-loop fuzzy logic transmission power control.

$\$$ Closed-loop fuzzy logic transmission power control.

!Adaptive multichannel.

under open-loop and closed-loop FLC configurations. Up to $40.62 \%$ and $38.6 \%$ energy savings were obtained by implementing the open-loop and closed-loop FLC-based TPC, respectively, relative to a fixed transmitted power (i.e., no TPC techniques). The study of [22] presented an efficient TPC. The control packet overhead was reduced using the measured temperature to control the transmitted power of SNs. The authors presented a new TPC technique that maintains the link quality as the temperature changes. A combination of S/W duty cycle and TPC was introduced by $[23,24]$. In a study [24], the energy-harvesting status and the remaining energy level were utilized to control the transmitted power of SNs. Thus, the lifespan of the entire WSN was extended, and the energy usage was balanced. By contrast, [23] computed the RSSI in the end device to control its transmitted power when the communication link broke. Energy savings of 26\% relative to a fixed transmitted power policy were obtained by adopting S/W and TPC techniques. Kim et al. [25] used the RSSI and link quality indicator (LQI) of a ZigBee body area network to control the level of transmitted power of SNs. The proposed technique, namely, RSSI/LQI-based TPC, effectively distinguished between signal attenuation and interference. When interference was unavailable in the link, the proposed method controlled the SN transmitted power based on the RSSI. Otherwise, the proposed method changed the present channel to avoid interference. The proposed method showed improvements in energy efficiency of up to $51.63 \%$ in the presence of interference.

In [26], the RSSI and packet reception rate (PRR) for communication link estimation were utilized to achieve adaptive multichannel transmission power control (AMC-TPC). The proposed technique was applied in an industrial application to reduce the power consumption of ZigBee WSNs, adapt to changing environmental situations, and adjust the transmission power level to overcome channel fading. The proposed technique can reduce the $\mathrm{SN}$ energy requirements by over $50 \%$. Finally, in [27], two TPC techniques were introduced to improve the energy consumption of the mobile node at different distances for outdoor and indoor environments. The two techniques were applied in practice using the ZigBee wireless protocol to show the WSN efficiency. $57 \%$ power savings were obtained relative to a fixed transmitted power. Six of the above state-of-the-art methods can be compared in terms of power savings, as summarized in Table 1. All these studies used a TPC technique as a power reduction technique to reduce the power consumption of sensor nodes in WSNs.

In contrast to existing research that solely used TPC or combined TPC with another technique to reduce the power consumption of SNs, this paper proposes three power consumption techniques for reducing the power consumption of mobile nodes. To the best of our knowledge, this combination of three techniques has not been adopted in previous studies. This paper proposes a TPC-based distance estimation algorithm that can be combined with three techniques which are (i) TPC technique, (ii) S/W strategy, and (iii) data rate. The state-of-the-art TPC technique is based on the RSSI. When the RSSI value exceeds the target threshold level, the communication link quality is good. In this case, the target decreases its transmitted power. In contrast, if the RSSI value falls below the target threshold level, the target increases its transmitted power because the communication link quality is poor. Otherwise, the target maintains its transmitted power. However, depending on the RSSI values, the distance estimation remains poor in an indoor environment when channel interference exists $[25,28]$. In this research, to overcome this problem, three anchor nodes were deployed in the track cycling field, and the RSSI values of these anchor nodes were used to train and test the ANN to accurately determine the distance between the bicycle that is moving on the cycle track and the coach. The major problem in current TPC techniques is the transmission of more control packets. Therefore, in this paper, a novel algorithm (i.e., a TPC-based accelerometer) is adopted to reduce data communication by dispensing the beacon signals, thereby minimizing the data overhead. Consequently, the mobile and master node power consumption can be significantly reduced. Moreover, both TPC-based distance and TPC-based accelerometer algorithms are adopted for outdoor and indoor velodromes.

\section{System Model}

The proposed WSN topology is shown in Figure 1. The topology consists of static and sensor nodes. The static nodes consist of three anchor nodes, namely, AN1, AN2, and AN3. AN1, which is located at the center of the track cycling field, handles the reception of the cyclist and bicycle parameters and sending a beacon signal to the $\mathrm{SN}$ for the distance 


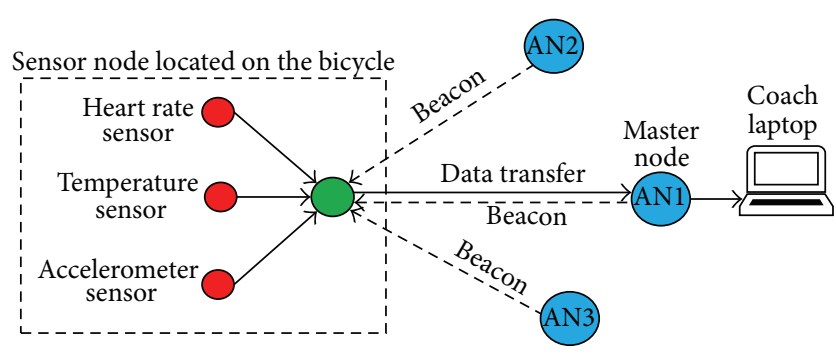

FIgURE 1: Entire WSN network topology.

estimation of the bicycle on the track. The received data by AN1 are displayed on the coach's laptop to allow the coach to monitor the cyclist's performance. AN1 is connected to the coach's laptop, thus requiring no battery source. The anchor nodes AN2 and AN3 are $27 \mathrm{~m}$ away from AN1, and their energy is derived from their individual power supplies. This means that $\mathrm{AN} 2$ and $\mathrm{AN} 3$ can be powered from the main $\mathrm{AC}$ source. Both AN2 and AN3 are not connected to AN1, but they are operating independently. The function of AN2 and AN3 is to send a beacon signal to the mobile node (bicycle) for distance estimation purposes.

The SN includes three sensors: a heart rate sensor for measuring the cyclist's heart rate during cycling, a temperature sensor for measuring the cyclist's temperature during cycling, and an accelerometer sensor for measuring the inclination angle of the bicycle on the track. Angle measurements can be used to inform the coach about the inclination angle of the cyclist on the track. This approach will allow the coach to know the section site of the cyclist during cycling on the track cycling field. In addition, the angle measurement provides a significant advantage for controlling the transmitted power and data rate of the SN when the TPC-based accelerometer algorithm is considered. The entire data packet structure of the $\mathrm{SN}$ based on the nRF24L01 wireless protocol is shown in Figure 2. Figure 2(a) is the general data packet frame of the nRF24L01 wireless protocol, whereas Figure 2(b) is a detailed version for this research. The ANT data packet structure of the SN consists of nine bytes: the identification node address ID (three bytes: one byte for each sensor), sensing data (five bytes: two bytes for heart rate, one byte for temperature, and two bytes for inclination angle), and the battery status (one byte).

The SN transmits the data packet to AN1 by combining all the data from the sensors into one data packet frame. Moreover, the SN receives a beacon frame consisting of one data byte from AN1 for distance estimation purposes. AN1 is called the master node, while the SN is known as the mobile node because the master node receives all the information of the $\mathrm{SN}$, whereas the $\mathrm{SN}$ is installed on and moves with the bicycle on the track.

\section{Methodology}

4.1. Wireless Channel Model. Most wireless channel models are derived based on a combination of practical and analytical methods. The practical method is based on curve fitting, whereas the analytical method depends on measured data. This approach has the advantage of being able to consider the tacit propagation factors in the channel through the actual field measurements [29]. Most wireless modules support RSSI, which allows the received power to be calculated for each received packet. The energy or power of a signal traveling between two nodes (i.e., mobile bicycle node and master node in our application) is a signal parameter that includes information reflecting the distance between those nodes. This parameter can be used along with LNSM and path loss for distance estimation [30]. Therefore, LNSM is given as $[31,32]$

$$
\mathrm{PL}(d) \mathrm{dBm}=\mathrm{PL}_{0}\left(d_{0}\right)+10 n \log _{10}\left(\frac{d}{d_{0}}\right)+X_{\sigma},
$$

where $\mathrm{PL}(d) \mathrm{dBm}$ is the reference path loss at a distance $d$ in meters on the track cycling and $\mathrm{PL}_{0}\left(d_{0}\right)$ is the path loss at a reference distance $d_{0}$. The path loss $\mathrm{PL}_{0}\left(d_{0}\right)$ can be obtained through field measurements or calculated using the Friis equation, where $n$ is a path loss exponent that indicates the rate at which the RSSI decreases with distance and $d$ is the distance between the master and mobile nodes in meters. The distance varies with the bicycle's location on the track, and $X_{\sigma}$ is a zero-mean Gaussian random variable (in decibels) with a standard deviation $\sigma$ (in decibels).

The RSSI at the mobile node can be calculated from the following:

$$
\text { RSSI }(\mathrm{dBm})=P_{t}(\mathrm{dBm})-\mathrm{PL}(d) \mathrm{dBm},
$$

where $P_{t}$ is the master node transmitter power in $\mathrm{dBm}$.

Then, the RSSI at the mobile node can be estimated from the following $[33,34]$ :

$$
\begin{aligned}
\operatorname{RSSI}(\mathrm{dBm})= & P_{t}(\mathrm{dBm})-\mathrm{PL}_{0}\left(d_{0}\right)-10 n \log _{10}\left(\frac{d}{d_{0}}\right) \\
& +X_{\sigma} .
\end{aligned}
$$

In this work, the parameter values of (3) were set as $\mathrm{PL}_{0}\left(d_{0}\right)=-37 \mathrm{dBm}$, which was obtained from the Friis equation for ANT module operating in the $2.4 \mathrm{GHz}$ ISM band; $d_{0}=1 \mathrm{~m}$ [19], where $d$ is the adopted velodrome distance ranging from $32 \mathrm{~m}$ to $65 \mathrm{~m} ; n=2.4[32,35]$ for outdoor velodrome; $n=3$ [36-38] for indoor velodrome; and $X_{\sigma}=4 \mathrm{~dB}[19,39]$ for both outdoor and indoor environments.

Equation (3) is used to generate the RSSI values (1,125 samples), which can be used for training and testing the ANN algorithm for distance estimation. Therefore, a hybrid PSOANN algorithm is used for distance estimation, as described in detail in Section 4.4.

4.2. ANT Current Consumption and Transmission Time Modeling. The average current consumption $I_{\text {avg }}$ for the ANT module of the mobile node can be computed using

$$
I_{\text {avg }}=\frac{I_{\text {active }} t_{\text {active }}+I_{\text {sleep }} t_{\text {sleep }}}{T_{\text {total }}},
$$




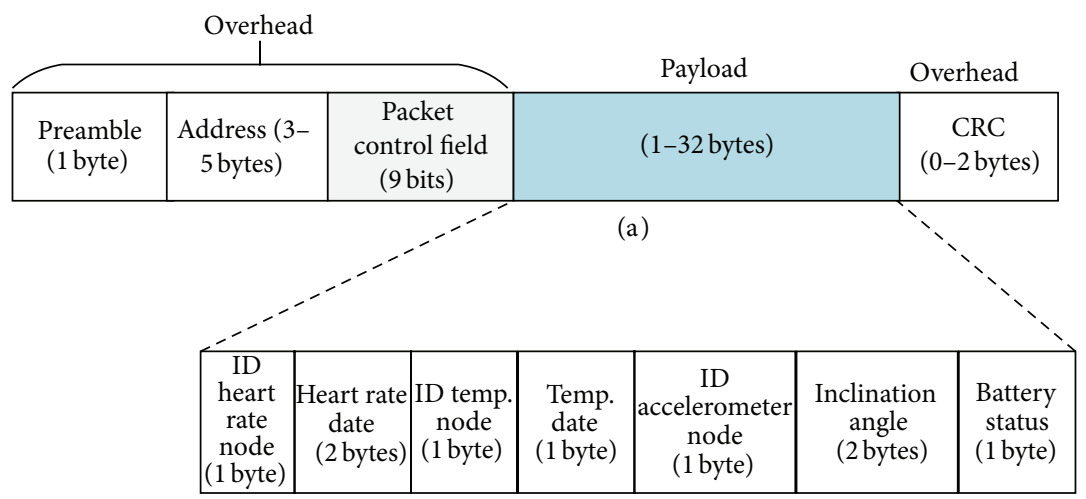

(b)

FIGURE 2: Data packet frame of the sensor node.

where $I_{\text {active }}$ and $t_{\text {active }}$ are the ANT active current and active transmission time, respectively; $I_{\text {sleep }}$ and $t_{\text {sleep }}$ are the ANT sleep current and sleep time, respectively; and $T_{\text {total }}$ is the time for one cycle (i.e., one second in this application).

$t_{\text {sleep }}=T_{\text {total }}-t_{\text {active }}$; therefore, (4) can be rearranged as

$$
I_{\text {avg }}=\frac{t_{\text {active }}}{T_{\text {total }}} I_{\text {active }}+\left(1-\frac{t_{\text {active }}}{T_{\text {total }}}\right) I_{\text {sleep }} \text {, }
$$

where $\left(t_{\text {active }} / T_{\text {total }}\right)$ is the duty cycle of the ANT protocol.

The active transmission time in (5) plays an important role in the power consumption of the mobile node. The power consumption can be reduced for short active transmission times because the active transmission time mainly depends on the data rate of the ANT. Therefore, deriving a mathematical relationship between the current consumption and the data rate is crucial for this application. The active transmission time in the enhanced ShockBurst ANT transmission mode can be expressed as follows [40]:

$$
t_{\text {active }}=\frac{L_{\mathrm{pl}}}{R_{\mathrm{SPI}}}+2 \frac{L_{\mathrm{p}}}{R}+T_{\mathrm{IRQ}}+2 T_{\mathrm{S} / \mathrm{A}},
$$

where $R$ is the ANT data rate, which the adjustment is limited to $250 \mathrm{kbps}, 1 \mathrm{Mbps}$, and $2 \mathrm{Mbps}$ by the manufacturer, and $L_{\mathrm{pl}}$ is the payload of the data packet. The maximum payload is 32 bytes [41], as shown for the packet length in Figure 2(a). In this work, the payload data for heart rate, temperature, and inclination angle transmitted from the mobile node to the master node consist of 9 bytes, as shown in Figure 2(b). The payload of the ANT is configurable (1-32 bytes), as mentioned above in the advantages of the ANT. Therefore, only 9 bytes as the payload out of 32 bytes will be sent. In that case, the payload size will be only 9 bytes. Therefore, the energy savings of the ANT wireless protocol will be improved. Thus, the payload is $(9 \times 8$ bits/byte $=72$ bits $)$; $R_{\mathrm{SPI}}$ is the data rate, namely, $10 \mathrm{Mbps}$, of the SPI interfacing between the ANT module and microcontroller; $L_{\mathrm{p}}$ is the ANT packet length, namely, 19 bytes ([9 payloads +8 headers $] \times 8$ bits $/$ byte +9 -bit control field $=145$ bits); $T_{\text {IRQ }}$ is the interruption time, namely,
6 and $8.2 \mu$ s for data rates of $2 \mathrm{Mbps}$ and $1 \mathrm{Mbps}$, respectively; and $T_{\mathrm{S} / \mathrm{A}}$ is the transition time from sleep to active modes. The transition time is from sleep to standby modes ( $150 \mu \mathrm{s}$ for using an external clock) and from standby to active modes $(130 \mu \mathrm{s})$, as in the nRF24L01 data sheet [42]. Thus, the total transition time from sleep mode to active mode and vice versa is $280 \mu \mathrm{s}$.

Equation (7) can be obtained by substituting the preceding values in (6):

$$
t_{\text {active }}=575.4 \times 10^{-6}+\frac{290 \text { bits }}{R} \text { in seconds. }
$$

According to (7), the active time ( $\left.t_{\text {active }}\right)$ is $1.735 \mathrm{~ms}, 865.4 \mu \mathrm{s}$, and $720.4 \mu \mathrm{s}$ for $250 \mathrm{kbps}, 1 \mathrm{Mbps}$, and $2 \mathrm{Mbps}$, respectively. Figure 3(a) illustrates the duty cycle for a $250 \mathrm{kbps}$ data rate for the mobile node at the transmission (Tx) mode and the master node at the receiver $(\mathrm{Rx})$ mode.

Consequently, (7) is substituted into (5). The average current consumption $\left(I_{\mathrm{avg}}\right)$ of the ANT for the mobile node in the Tx mode and the master nodes in the Rx mode can be expressed as $I_{\text {avgl }}$ and is computed based on the following:

$$
\begin{aligned}
I_{\mathrm{avg} 1}= & \frac{575.4 \times 10^{-6}+290 \mathrm{bits} / R}{T_{\text {total }}} I_{\text {active }} \\
& +\left(1-\frac{575.4 \times 10^{-6}+290 \mathrm{bits} / R}{T_{\text {total }}}\right) I_{\text {sleep }}
\end{aligned}
$$

Equation (8) shows that the current consumption of the mobile node primarily depends on the ANT data rate and total time $T_{\text {total }}$, where $T_{\text {total }}$ is constant in this work (i.e., one second was adopted). The current consumption of the mobile node decreases when the data rate increases and vice versa. For the beacon's signal, that is, the master node in the Tx mode and the mobile node in the Rx mode, transmitting one byte of payload data is sufficient to determine the RSSI signal by the mobile node. In this case, the active time and the average current consumption of the ANT for the master node in the Tx mode and the mobile node in the Rx mode 


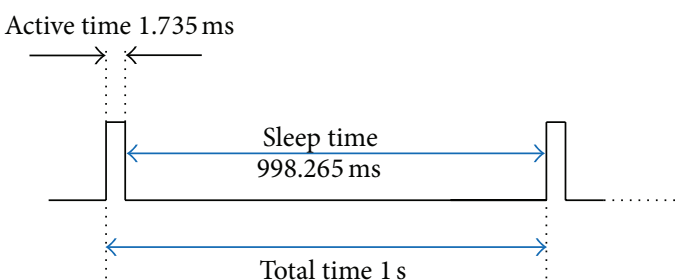

(a)

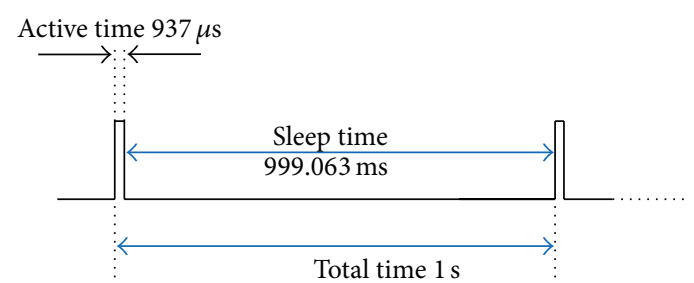

(b)

Figure 3: The duty cycle for the $250 \mathrm{kbps}$ data rate for (a) the mobile node in the Tx mode and the master node in the Rx mode and (b) the master node in the Tx mode and the mobile node in Rx mode.

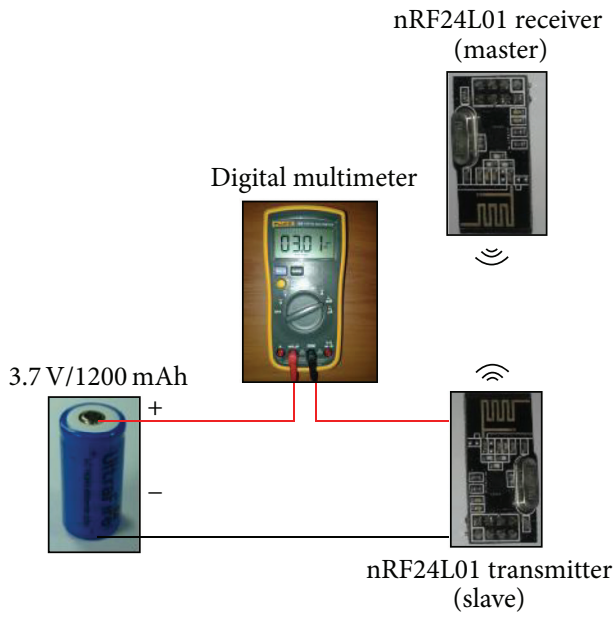

(a)

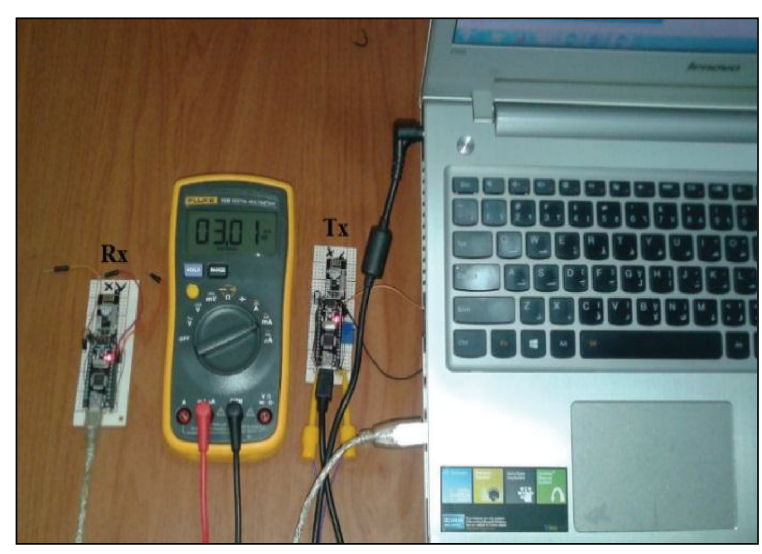

(b)

FIGURE 4: Testbed for the nRF24L01 current consumption measurements: (a) schematic diagram and (b) snapshot of the measurement.

can be expressed as $I_{\mathrm{avg} 2}$ and are computed as in the following equations:

$$
\begin{aligned}
t_{\text {active }}= & 289 \times 10^{-6}+\frac{162 \text { bits }}{R} \text { in seconds, } \\
I_{\text {avg } 2}= & \frac{289 \times 10^{-6}+162 \text { bits } / R}{T_{\text {total }}} I_{\text {active }} \\
& +\left(1-\frac{289 \times 10^{-6}+162 \text { bits } / R}{T_{\text {total }}}\right) I_{\text {sleep }} .
\end{aligned}
$$

Consequently, the active time ( $\left.t_{\text {active }}\right)$ is 937,451 , and $370 \mu \mathrm{s}$ for $250 \mathrm{kbps}, 1 \mathrm{Mbps}$, and $2 \mathrm{Mbps}$, respectively. Figure 3(b) illustrates the duty cycle for the $250 \mathrm{kbps}$ data rate for the master node in the Tx mode and the mobile node in the $\mathrm{Rx}$ mode.

4.3. ANT Current Consumption Measurements. The active and sleep current consumption of the ANT module can be measured using a digital multimeter (FLUKE 15B digital multimeter with a current measurement accuracy of $\pm 1.5 \%$ ) as shown in the diagram in Figure 4(a). The current consumption is measured during the transmission process when the ANT mobile node communicates with the ANT master node. For this purpose, the digital multimeter is connected in series between the supply line of the ANT module and a 3.7 V/1200 mAh LiPo rechargeable battery. The multimeter measures the active and sleep currents consumed by the ANT module on the order of milliamperes and microamperes, respectively. The active, sleep, and average current consumption of the ANT module in both the Tx and $\mathrm{Rx}$ modes are obtained at different $\mathrm{RF}$ transmission powers $\left(P_{\mathrm{Tx}}\right)$ and data rates, as shown in Tables 2 and 3 . The active and sleep current consumption were obtained from the testbed measurements, as shown in Figure 4(b). By contrast, the average current consumption for the ANT in the Tx and $\mathrm{Rx}$ modes is computed based on (8) and (10). The values in Table 2 can be considered for the mobile node and master nodes in the $\mathrm{Tx}$ and $\mathrm{Rx}$ modes, respectively, whereas the values for the master and mobile nodes in the Tx and $\mathrm{Rx}$ modes can be found in Table 3 , respectively.

4.4. Adopted Distance Estimation Technique. Artificial neural networks (ANNs) can represent the complex correlations between output and input variables, and the corresponding relationship can be directly formed from the data. Nonlinear data can be processed to estimate the locations or distances of SNs in the network. ANNs have been proposed in several 


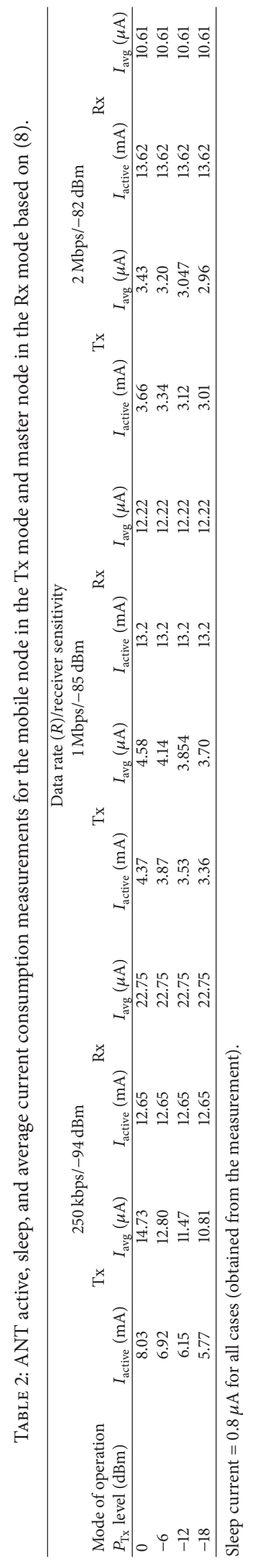




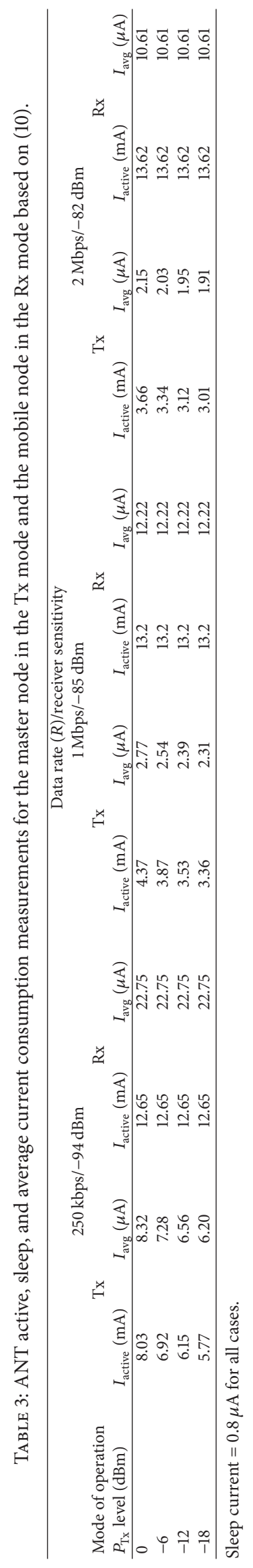


TABLE 4: Parameters of the mobile node in different velodrome sections (TPC-based distance estimation algorithm).

\begin{tabular}{|c|c|c|c|c|c|c|}
\hline \multirow{2}{*}{ Parameter } & \multicolumn{2}{|c|}{ Section 1} & \multicolumn{2}{|c|}{ Section 2} & \multicolumn{2}{|c|}{ Section 3} \\
\hline & Outdoor & Indoor & Outdoor & Indoor & Outdoor & Indoor \\
\hline Distance between mobile node and coach (m) & $32-46$ & $32-46$ & $46-54$ & $46-54$ & $54-65$ & $54-65$ \\
\hline Transmitted power $\left(P_{\mathrm{Tx}}\right)$ of mobile node $(\mathrm{dBm})$ & -6 & -6 & 0 & -6 & 0 & 0 \\
\hline Data rate (bps) & $2 \mathrm{M}$ & $250 \mathrm{k}$ & $2 \mathrm{M}$ & $250 \mathrm{k}$ & $2 \mathrm{M}$ & $250 \mathrm{k}$ \\
\hline Tx mode $I_{\text {active }}(\mathrm{mA})$ & 3.34 & 6.92 & 3.66 & 6.92 & 3.66 & 8.03 \\
\hline Rx mode $I_{\text {active }}(\mathrm{mA})$ & 13.62 & 12.65 & 13.62 & 12.65 & 13.62 & 12.65 \\
\hline$\left(\mathrm{Tx} \_I_{\text {active }}+\mathrm{Rx} \_I_{\text {active }}\right) / 2$ & 8.48 & 9.785 & 8.64 & 9.785 & 8.64 & 10.34 \\
\hline
\end{tabular}

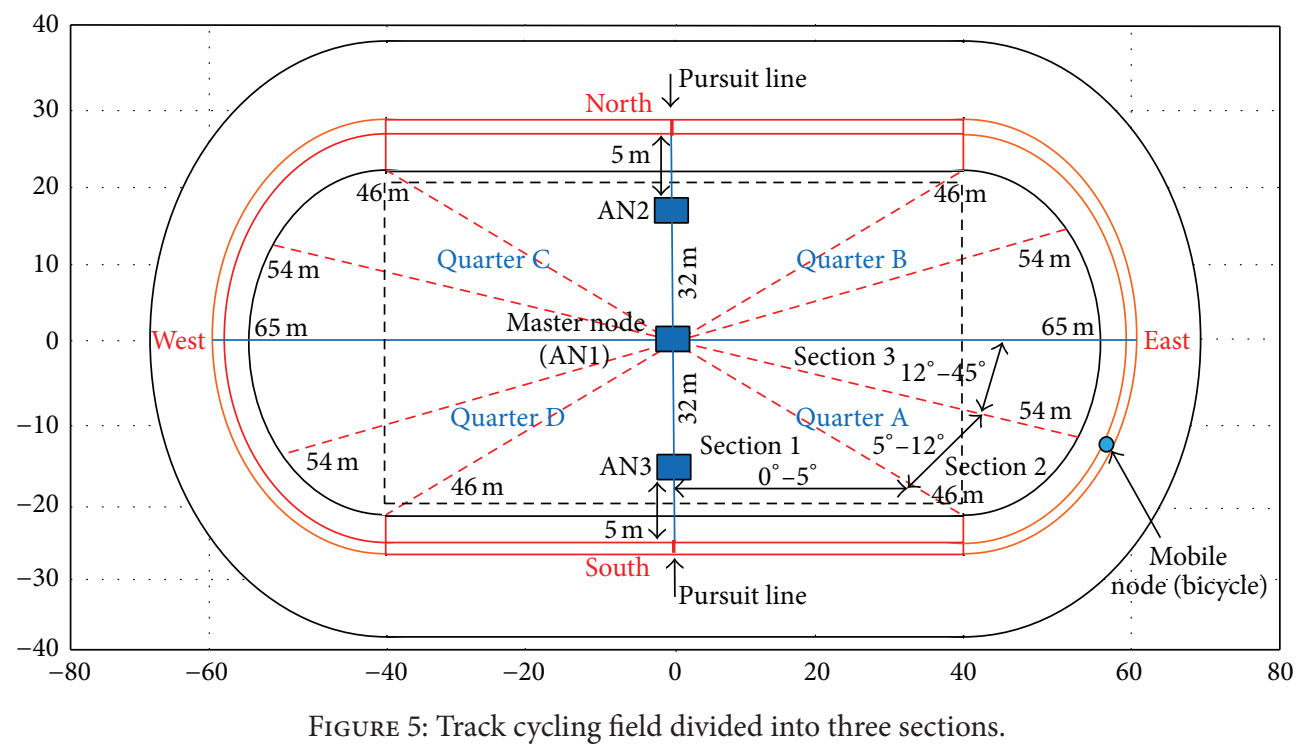

studies to localize the nodes of a WSN [43,44]. In the current paper, a feed-forward neural network trained with a Levenberg-Marquardt (LM) algorithm was considered to estimate the distance between the mobile node and the master node. The LM training algorithm was considered in this work because it provides a minimum distance error, as proven in [43], as well as because of its speed and efficiency. The RSSI values at three anchor nodes (AN1, AN2, and AN3), which were received by the mobile node, were used as the input matrix to the ANN, whereas the output value of the ANN is the actual distance between the mobile node and the master node. With both the input and output sets, the ANN was trained and tested on the data. Particle swarm optimization (PSO) improved the performance of the ANN in selecting the optimum values of the number of nodes in each hidden adopted layer and the learning rate of the ANN, as in Algorithm 1 (see Algorithm 1 in Supplementary Material available online at http://dx.doi.org/10.1155/2016/ 7314207). The proposed hybrid PSO-ANN algorithm reduced the distance estimation error. In this paper, the distances between the mobile node on the bicycle track and the master node at the outdoor and indoor velodromes were estimated. Exactly 375 samples of RSSI values at each anchor node were generated based on the LNSM. In total, 1,125 samples were collected from the three anchor nodes by the mobile node to estimate the distance between the mobile node and the master node.

4.5. Estimation of Optimal RF Transmitted Power and Data Rate. The velodromes can each be divided into four symmetrical quarters; each quarter can be divided into three sections according to the distance and the inclination angle of the bicycle on the track. The distances between the mobile node and the master node can be divided into three sections, namely, (i) Section 1 (32-46 m), (ii) Section 2 (46-54 m), and (iii) Section $3(54-65 \mathrm{~m})$, as shown in Figure 5. The division of the velodromes was based on the metric values of the transmission power, data rate, and current consumption of the mobile node; these values are shown in a mapping table (see Table 4). These values were selected to obtain the minimum power consumption and to ensure the data communication between the mobile node and the master node. Through a MATLAB simulation, Figure 6 was obtained and shows the received power at the master node from the mobile node in each section. The received power is made greater than the master node receiver sensitivity in each section to ensure the communication link, as shown in Figure 6. For outdoor velodromes, in Sections 1, 2, and 3, the received power by the master node was greater than its sensitivity thresholds (i.e., $-82,-85$, and $-94 \mathrm{dBm}$ ). Therefore, the data rate of 
TABLE 5: Parameters of the mobile node in different velodrome sections (TPC-based accelerometer algorithm).

\begin{tabular}{|c|c|c|c|c|c|c|}
\hline \multirow{2}{*}{ Parameter } & \multicolumn{2}{|c|}{ Section 1} & \multicolumn{2}{|c|}{ Section 2} & \multicolumn{2}{|c|}{ Section 3} \\
\hline & Outdoor & Indoor & Outdoor & Indoor & Outdoor & Indoor \\
\hline Inclination angle of the bicycle on the track (degree) & $0-5^{\circ}$ & $0-5^{\circ}$ & $5^{\circ}-15^{\circ}$ & $5^{\circ}-15^{\circ}$ & $>15^{\circ}$ & $>15^{\circ}$ \\
\hline Transmitted power of the mobile node $\left(P_{\mathrm{Tx}}\right)(\mathrm{dBm})$ & -6 & -6 & 0 & -6 & 0 & 0 \\
\hline Data rate (bps) & $2 \mathrm{M}$ & $250 \mathrm{k}$ & $2 \mathrm{M}$ & $250 \mathrm{k}$ & $2 \mathrm{M}$ & $250 \mathrm{k}$ \\
\hline Tx mode $I_{\text {active }}(\mathrm{mA})$ & 3.34 & 6.92 & 3.66 & 6.92 & 3.66 & 8.03 \\
\hline
\end{tabular}

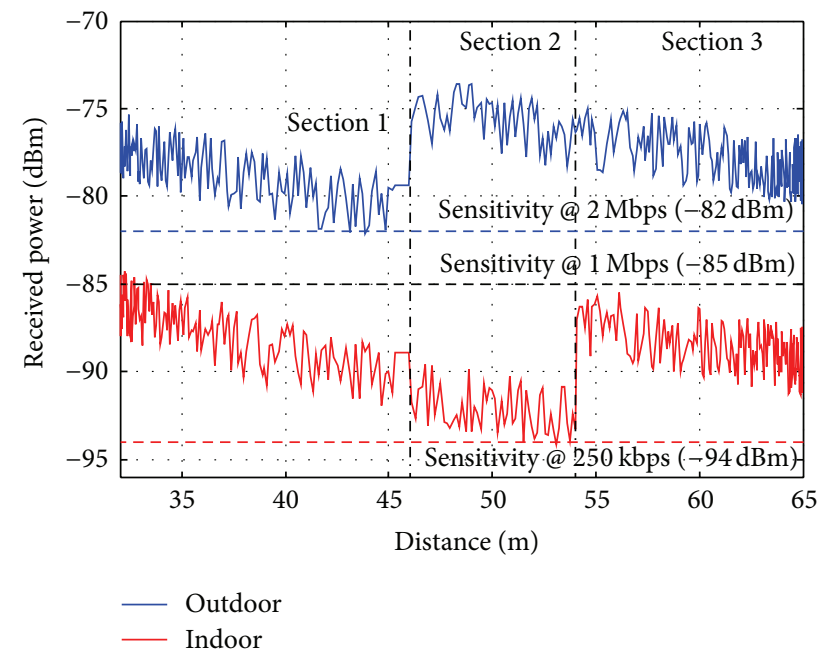

FIGURE 6: Received power by the master node with respect to the distance on the track.

the mobile node can be configured to 2 Mbps to reduce its power consumption. In contrast, for indoor velodromes, the received power by the master node fell below the master node sensitivity thresholds (i.e., -82 and $-85 \mathrm{dBm}$ ) and was greater than $-94 \mathrm{dBm}$ for Sections 1,2 , and 3. Therefore, the data rate of the mobile node can be configured to $250 \mathrm{kbps}$ to ensure data communication with the master node.

Similarly, the inclination angles of the bicycle while moving on the track can be divided into three sections, namely, (i) Section $1\left(0^{\circ}-5^{\circ}\right)$, (ii) Section $2\left(5^{\circ}-12^{\circ}\right)$, and (iii) Section $3\left(12^{\circ}-45^{\circ}\right)$. This division was set according to the nature of the velodromes: it is flat in Section 1, it is slightly sloping in Section 2, and it has a high inclination angle in Section 3. Consequently, a relationship between the transmitted power, data rate, and current consumption can be established, as illustrated in a mapping table (see Table 5).

AN2 and AN3 were fixed at the north and south side of the track, respectively, to ensure the communication link with the mobile node on the cycle track. The farthest distance from the mobile node becomes $65 \mathrm{~m}$. In contrast, AN2 and AN3 cannot be located at the east or west side because the mobile node on the track will be at the farthest distance (i.e., $130 \mathrm{~m}$ ) from them. Consequently, the communication link will be lost.

4.6. TPC-Based Distance Estimation Algorithm. Transmission power control (TPC) is an essential concern in the design of an energy-efficient WSN. TPC has been used in various WSN applications $[18,19,23,26]$. In WSNs, the TPC can be used with a mobile SN to save energy and maintain network connectivity. In our application, the transmitted power, duty cycle, and data rate of the mobile node, which moves on the track in a regular path, were investigated as a function of the estimated distance between the mobile node and the master node. The distance was accurately determined based on the hybrid PSO-ANN algorithm, which was presented in Section 4.4.

The TPC and the data rate adjustment of the ANT improved the power consumption at the physical layer. To further reduce the power consumption, the sleep/wake strategy was applied at the Media Access Control (MAC) layer for the RF module to schedule sleep. The TPC can be combined with the sleep/wake algorithm (TPCS/W) and data rate adjustment to significantly improve the power savings of the ANT. Once the mobile node approaches the master node, the mobile node operates at a low transmission power level and data rate. By contrast, the mobile node operates at a high transmission power level and data rate when it moves away from the master node location to maintain the communication connectivity. Algorithm 2 (given in Supplementary Material) shows the adjusting conditions of the TPC technique for the outdoor velodrome. For the indoor velodrome, the same algorithm (i.e., Algorithm 2) can be applied but with changes to the values of the transmitted power, data rate, and current consumption, as illustrated in Table 4. The TPC technique has an additional advantage of reducing the risk of interference in the communication channel; that is, the interference decreases when the transmission power decreases [45].

4.7. TPC-Based Accelerometer Algorithm. The transmission power and data rate of the ANT mobile node can be adjusted based on a novel algorithm that adopts the sensing of the inclination angles of the bicycle as it moves along the track. For this purpose, the microelectromechanical system ADXL 335 accelerometer was considered. The ADXL335 is a lowpower, three-axis $(x, y$, and $z$ ) accelerometer based on capacitive transduction principles. It can measure acceleration on a full-scale range of $\pm 3 \mathrm{~g}$ [46]. It has a sensitivity of $300 \mathrm{mV} / \mathrm{g}$, offset fluctuations of less than $\pm 1 \mathrm{mg} /{ }^{\circ} \mathrm{C}$, and high noise performance, thus permitting a high-quality output signal [47]. The bandwidth of the ADXL335 is $1600 \mathrm{~Hz}$ for the $x$ and $y$-axes and $550 \mathrm{~Hz}$ for the $z$-axis [48]. The accelerometer is compact and lightweight [49]. The output voltage along each axis is proportional to the acceleration along the specific 
axis. The supply voltage of the ADXL335 is $1.8-3.6 \mathrm{~V}$. In this application, the accelerometer can be provided with $+3.3 \mathrm{~V}$ to provide the supply voltage of the Atmega328p microcontroller of the mobile node. With this supply voltage, the ADXL335 consumes $270 \mu \mathrm{A}$ during sensing events (obtained through measurement with a FLUKE 15B digital multimeter).

In this application, one triple-axis ADXL335 accelerometer will be attached to the bicycle frame to measure the inclination angles as the bike moves along the track. The three adopted velodrome sections can be employed for the same purpose of TPC. However, the sections here were divided according to the measured angles instead of the distances; these sections were discussed in Section 4.5. In the TPCbased accelerometer algorithm, the values of the parameters, which are presented in Table 5, can be set to enable power savings in the mobile node. The values of the transmitted power and data rate are configured based on the same three sections for the TPC-based distance estimation algorithm. Therefore, Algorithm 3 (given in Supplementary Material) can be applied to the outdoor velodrome or environment. The same algorithm can be used for the indoor velodrome or environment but with different values of the transmitted power, data rate, and current consumption, as shown in Table 5. However, one of the major advantages of the TPCbased accelerometer algorithm is its capacity to reduce the power consumption of both the mobile node and the master node, thereby reducing the total power consumption of the WSN.

The other advantages of the TPC-based accelerometer algorithm over the TPC-based distance estimation algorithm are as follows. (i) The TPC-based accelerometer algorithm modifies the transmitted power and data rate of the ANT (self-healing) of the mobile node when the cyclist is on the track. Thus, the transmission of frequent beacon signals from the master node to the mobile node is not required. Consequently, the master and mobile nodes will obtain reduced energy consumption. By contrast, in the TPC-based distance estimation algorithm, the master node transmits the beacon signals at the maximum power (i.e., $0 \mathrm{dBm}$ and a high data rate of $250 \mathrm{kbps}$ ) to ensure the communication connectivity between the two nodes, causing high dissipated power in the master and mobile nodes. (ii) The master node can enter sleep mode by scheduling sleep after the transmitted data of the mobile node are received to reduce its power consumption, thereby further reducing the total power consumption of the WSN. (iii) A smaller memory size is required in the mobile node relative to the memory requirement of the TPC-based distance estimation algorithm, where the ANN requires a considerable amount of working memory [50]. The smaller memory requirement of the TPC-based accelerometer algorithm also reduces the size and complexity of the mobile node. (iv) The TPCbased accelerometer algorithm decreases the computational capacity of the processor of the mobile node. By contrast, in the TPC-based distance estimation algorithm, the ANN performs intensive computations to produce the distance estimation. Thus, both the time and power requirements are reduced in the TPC-based accelerometer algorithm. (v) The TPC-based accelerometer algorithm is more accurate than the TPC-based distance estimation algorithm. The distance estimated by the TPC-based distance estimation algorithm is based on RSSI values. However, the RSSI is subject to path loss and is influenced by channel environments, which vary over time; consequently, the distance measurement accuracy will be affected by the propagation channel. In the TPCbased accelerometer algorithm, however, the measurement accuracy is based on the accelerometer sensor installed on the bike. (vi) Finally, the TPC-based accelerometer algorithm employs simplex communication, in which the mobile node functions as the transmitter and the master node functions as the receiver. By contrast, in TPC-based distance estimation algorithm, each of the mobile and master nodes functions as both transmitter and receiver, resulting in an increase in the overhead and consequently in the power that can be dissipated by both nodes.

\section{Simulation Results}

5.1. Hybrid PSO-ANN Algorithm for Distance Estimation. The RSSI values of the three anchor nodes, namely, AN1, AN2, and AN3, for both the outdoor and indoor velodromes are received by the mobile node, which moves along the track. 375 samples of RSSI values were collected in quarter A for each anchor node; in total, 1,125 samples were collected from the three anchor nodes by the mobile node, as shown in Figure 7. These RSSI values are used as input to the ANN. The PSO algorithm is employed to determine the optimum learning rate and the optimum number of neurons in each adopted hidden layer to improve the distance estimation accuracy. The PSO algorithm is executed for 20 swarms and 500 iterations. Consequently, the fitness function will be obtained, as shown in Figure 8. The figure shows that the fitness function of the PSO algorithm is approximately $0.031 \mathrm{~m}$ after 281 iterations. Based on the results of the hybrid PSO-ANN algorithm, the ANN parameters are listed in Table 6. The training processes of the ANN algorithm are repeated several times using 1,000 iterations, or epochs, until the error between the actual and estimated distances is minimized.

The performance of the ANN is calculated based on the mean absolute error (MAE), mean square error (MSE), and root mean square error (RMSE). To evaluate the performance of the hybrid PSO-ANN algorithm, the MAE, MSE, and RMSE are plotted for both outdoor and indoor environments, as shown in Figure 9. The obtained MAE, MSE, and RMSE are $0.0122,0.0012$, and 0.0348 , respectively, for the outdoor environment and $0.0913,0.0411$, and 0.2028 , respectively, for the indoor environment based on the hybrid PSO-ANN algorithm. Figure 10 shows a comparison of the proposed hybrid PSO-ANN algorithm with other artificial intelligent algorithms employed for outdoor and indoor wireless SN localization in other studies. The results reveal that the location estimated using the hybrid PSO-ANN algorithm outperforms the algorithms of previous studies $[33,51-65]$ in terms of MAE.

5.2. TPC-Based Distance Estimation Algorithm. The TPCbased distance estimation algorithm (Algorithm 2 ) results are 
TABLE 6: Designed parameters of the ANN based on the PSO algorithm.

\begin{tabular}{|c|c|c|}
\hline Parameters & Value & Type \\
\hline Number of inputs & 3 & RSSI \\
\hline Number of hidden layers & 2 & $\begin{array}{l}\text { Hyperbolic tangent sigmoid activation } \\
\text { function }\end{array}$ \\
\hline Number of outputs & 1 & Linear activation function/distance \\
\hline \multirow[t]{2}{*}{$\begin{array}{l}\text { Number of neurons in each hidden layer } \\
(N 1 \text { and N2) }\end{array}$} & 18 & $\begin{array}{l}\text { For } N 1 \text { (obtained from the PSO } \\
\text { algorithm) }\end{array}$ \\
\hline & 18 & $\begin{array}{l}\text { For N2 (obtained from the PSO } \\
\text { algorithm) }\end{array}$ \\
\hline Learning rate $\left(L_{R}\right)$ & 0.5941 & Obtained from the PSO algorithm \\
\hline Number of iterations & 1000 & \\
\hline Target error (goal) & $0.001 \mathrm{~m}$ & \\
\hline \multirow{2}{*}{ Number of collected data samples } & 1125 & RSSI samples for outdoor environment \\
\hline & 1125 & RSSI samples for indoor environment \\
\hline
\end{tabular}

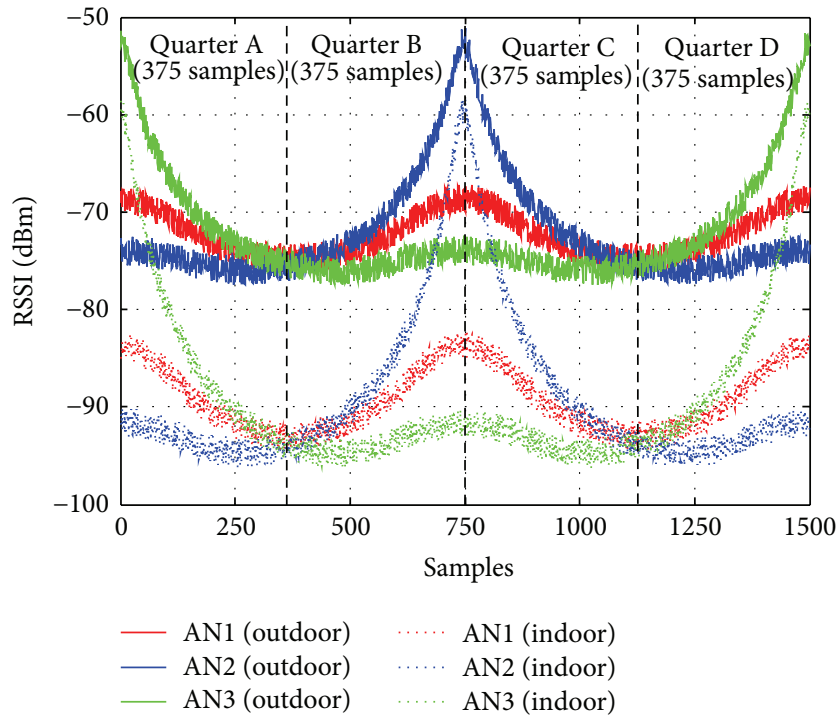

Figure 7: The RSSI values received by the mobile node in the outdoor and indoor velodromes.

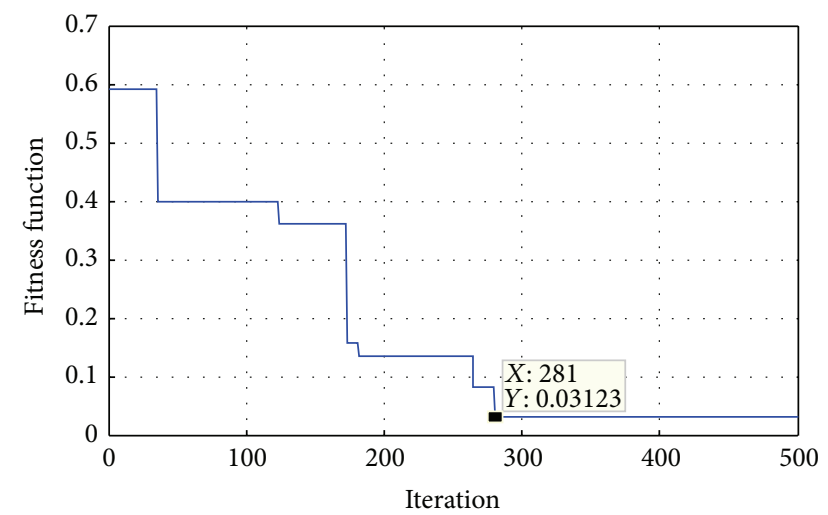

FIGURE 8: Fitness function versus iteration.

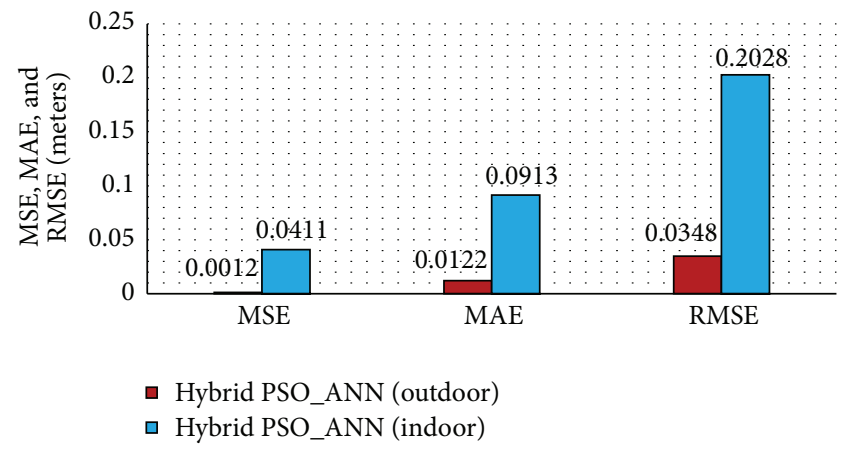

FIGURE 9: MSEs, MAEs, and RMSEs computed using the proposed hybrid PSO-ANN algorithm for the indoor and outdoor environments.

described in Sections 5.2.1 to 5.2.3. This section consists of three topics: the TPC technique, the sleep/wake strategy, and a combination of the TPC and sleep/wake strategy known as the TPCS/W technique. The results of these subsections were obtained using the MATLAB simulation software and are investigated and discussed in terms of power savings for each section of the cycle track in outdoor and indoor velodromes.

5.2.1. TPC Technique. When the TPC technique is solely applied to the ANT wireless protocol, the transmitted power and data rate are selected according to the three sections, as mapped in Table 4. Consequently, the current consumption in each section will be determined. As a result, Figures 11(a) and 11(b) are obtained for the outdoor and indoor velodromes, respectively. The two figures show the power savings in each section of the velodromes relative to the fixed transmitted power (i.e., $P_{\mathrm{Tx}}=0 \mathrm{dBm}$, data rate $=250 \mathrm{kbps}$, and the average current consumption of the mobile node in the Tx and Rx modes is $10.34 \mathrm{~mA}$ ).

In the outdoor velodrome, once the distance between the mobile node and the master node lies within Section 1, the 


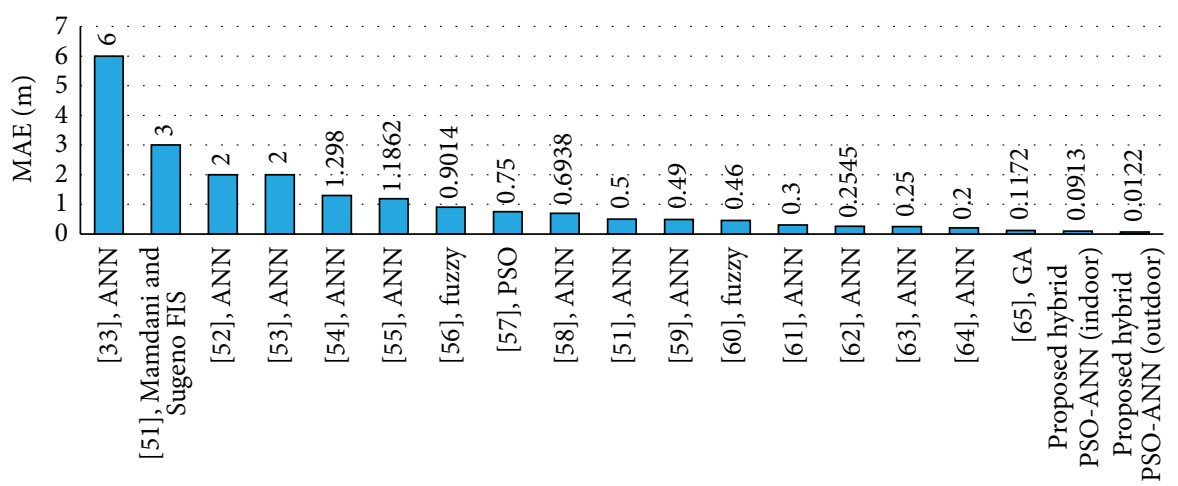

FIGURE 10: Comparison of the proposed hybrid PSO-ANN algorithm with other artificial intelligent algorithms employed in previous studies.

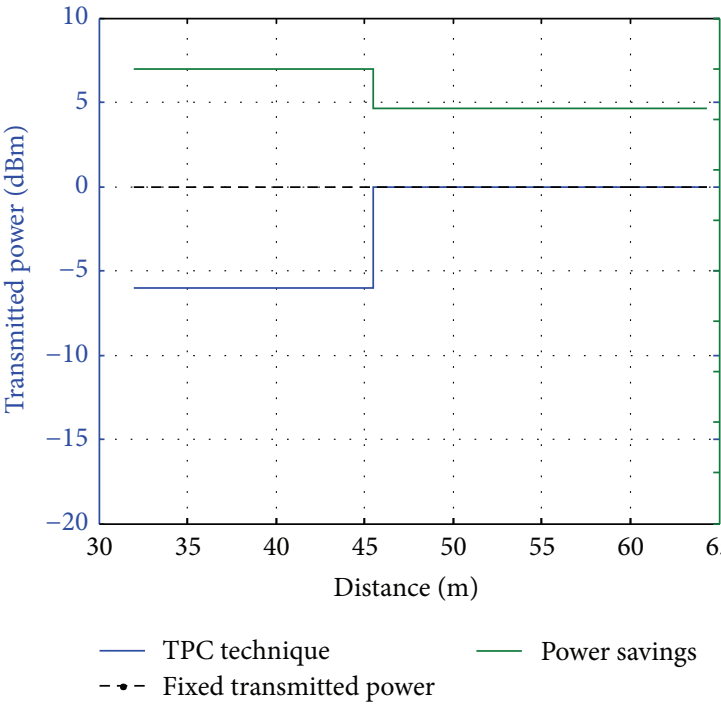

(a)

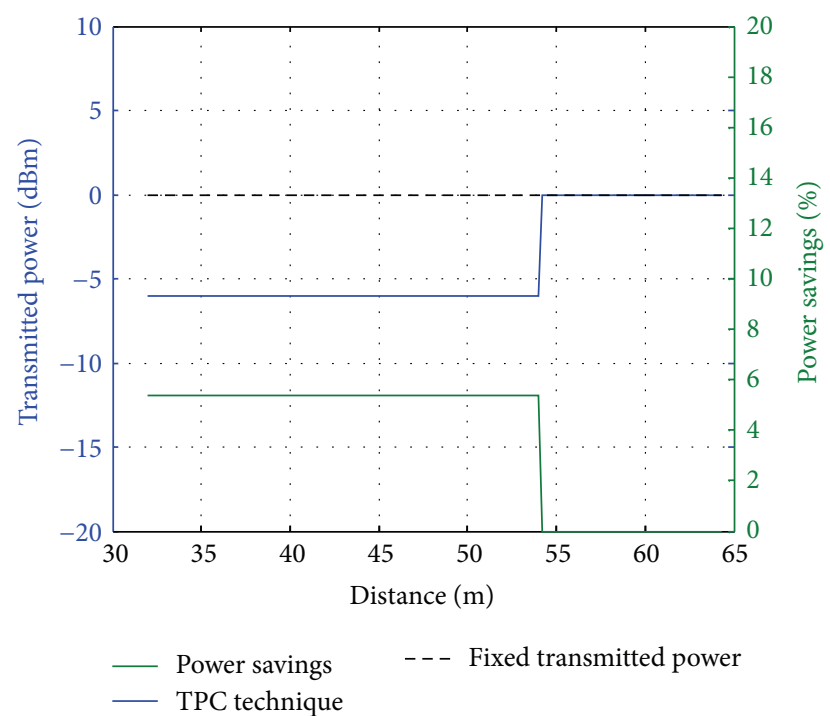

(b)

FIGURE 11: Transmitted power and power savings in (a) the outdoor velodrome and (b) the indoor velodrome obtained by applying the TPC technique.

ANT transmits the data at $-6 \mathrm{dBm}$ and a high data rate of $2 \mathrm{Mbps}$ to reduce the power consumption. When the distance between the master node and the mobile node lies within Sections 2 and 3 , the ANT sends the data at a high power level of $0 \mathrm{dBm}$ and a high data rate of $2 \mathrm{Mbps}$ to ensure data packet delivery. Therefore, more power can be saved in Section 1 than in Sections 2 and 3, as shown in Figure 11(a). The power savings in Section 1 are 18\%, whereas the power savings in Sections 2 and 3 are $16.44 \%$.

In the indoor velodrome, when the mobile node is moving within Sections 1 and 2, the transmitted power is $-6 \mathrm{dBm}$, and a minimum data rate of $250 \mathrm{kbps}$ is considered. These two values are selected in Sections 1 and 2 because such values result in the minimum power consumption, as previously shown in Table 4 . By contrast, the mobile node transmits at $0 \mathrm{dBm}$ and a data rate of $250 \mathrm{kbps}$ in Section 3 to ensure data packet delivery from the mobile node to the master node. Accordingly, the power savings in Sections 1 and 2 are $5.36 \%$ greater than in Section 3 (no power savings), as shown in Figure 11(b).

5.2.2. Sleep/Wake Strategy. A sleep/wake strategy can be applied to the ANT wireless protocol. In this work, a 1-second $\left(T_{\text {total }}\right)$ interval between consecutive transmissions of the sensed information is considered. The power consumption of the mobile node can be reduced in both the Tx and Rx modes. The mobile node is awake for a small fraction of the time and sleeps during the remainder of the total time. It simply wakes up to transmit data to or receive data from the master node. The average current consumption of the mobile node for outdoor and indoor velodromes was calculated using (8) for the Tx mode and using (10) for the Rx mode. The active and average current consumption of the mobile node in the Tx mode were $8.03 \mathrm{~mA}$ and $4.7 \mu \mathrm{A}$, respectively, whereas the active and average current consumption were $12.65 \mathrm{~mA}$ and $12.7 \mu \mathrm{A}$ in the $\mathrm{Rx}$ mode, respectively. Consequently, the power 


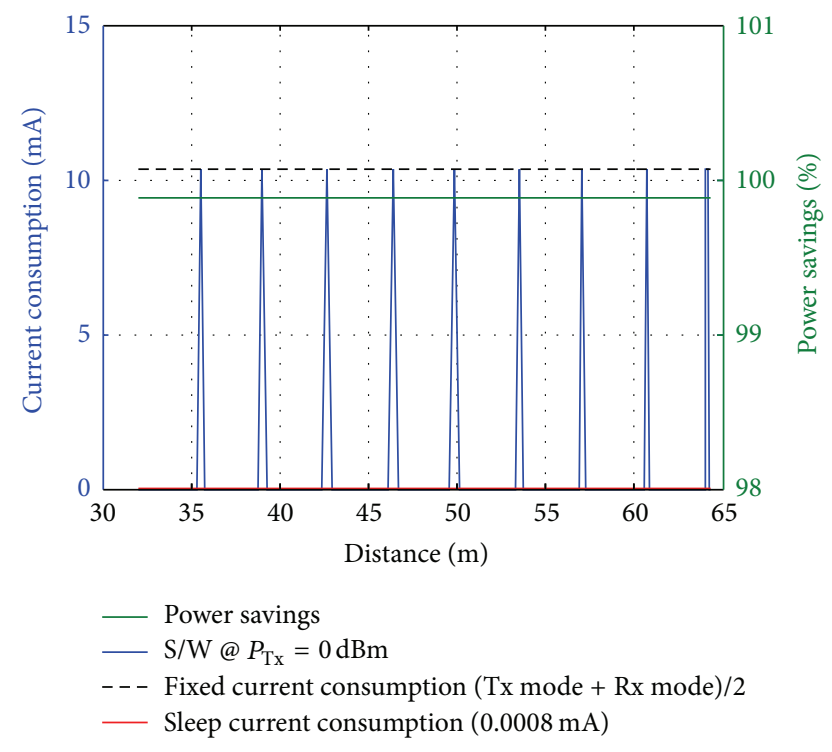

FIGURE 12: Current consumption in both outdoor and indoor velodromes using the sleep/wake strategy.

savings of the mobile node are $99.816 \%$ in the Tx mode and $99.9 \%$ in the Rx mode. Thus, the average power savings of the mobile node can be improved to $99.858 \%$ relative to the fixed current consumption in the Tx mode (i.e., $8.03 \mathrm{~mA}$ ) and the $\mathrm{Rx}$ mode (i.e., $12.65 \mathrm{~mA}$ ) for both outdoor and indoor velodromes, as shown in Figure 12.

5.2.3. TPCS/W Technique. When the TPCS/W technique is applied, the power savings in the outdoor velodrome slightly change between $99.9306 \%$ in Section 1 and $99.9316 \%$ in Sections 2 and 3 relative to the classical power consumption, as shown in Figure 13(a). By contrast, in the indoor velodrome, the power savings vary between $99.8575 \%$ in Sections 1 and 2 and 99.8582\% in Section 3, as shown in Figure 13(b). Figure 14 presents a comparison of the three adopted power-saving techniques, namely, the TPC technique, the sleep/wake strategy, and the TPCS/W technique. When the TPC technique is considered, the average ANT power savings improve by $16.96 \%$ (obtained from power savings in [Section $1+$ Section $2+$ Section 3]/3) in the outdoor velodrome and improve slightly by $3.57 \%$ in the indoor velodrome relative to the classical power consumption. Both the sleep/wake strategy and the TPCS/W technique significantly improve the average power savings of the mobile node by more than $99 \%$ in the outdoor and indoor velodromes, as shown by the bar chart in Figure 14 .

The results indicate that the power savings of the mobile node in the outdoor velodrome are higher than those in the indoor velodrome. Such a difference is attributed to the greater propagation path loss in the indoor velodrome, thereby requiring the mobile node in the indoor velodrome to transmit data at a higher power level and a lower data rate, particularly in Section 3 of the velodrome, to ensure data packet delivery from the mobile node to the master node. In addition, when the TPCW/S technique is applied, the power savings slightly improve relative to those when the sleep/wake strategy is applied and significantly improve relative to the classical power consumption. Therefore, only the TPC and $T P C S / W$ techniques are discussed in the subsequent section when the TPC-based accelerometer algorithm is considered.

5.3. TPC-Based Accelerometer Algorithm. The accelerometer ADXL335 can be employed to control the transmitted power and data rate of the ANT wireless protocol based on the bicycle inclination angle on the track. When the bike is moving on the track, the bicycle's inclination angle changes according to the nature of the velodrome, as shown in the simulation results in Figure 15. In the TPC-based accelerometer algorithm (Algorithm 3), the ANT wireless protocol functions only as the transmitter because the transmitted power and data rate are controlled based on the inclination angle and does not require a beacon signal. Thus, the power savings generated by the TPC-based accelerometer algorithm are expected to be better than those by the TPC-based distance estimation algorithm. Therefore, the power consumption of the mobile node is investigated in the following subsection.

5.3.1. TPC Technique. When the TPC technique is solely applied to the ANT wireless protocol, the transmitted power and data rate are selected based on the three velodrome sections, as mapped in Table 5. Consequently, the current consumption in each section is determined. As a result, Figures 16(a) and 16(b) will be obtained for the outdoor and indoor velodromes, respectively. The two figures demonstrate the power savings in each section of the velodromes relative to the fixed transmitted power (i.e., $P_{\mathrm{Tx}}=0 \mathrm{dBm}$, data rate $=$ $250 \mathrm{kbps}$, and current consumption $=8.03 \mathrm{~mA}$ ). In the outdoor velodrome, the power savings are $58.4 \%$ in Section 1 and 54.42\% in Sections 2 and 3, as shown in Figure 16(a). By contrast, in the indoor velodrome, the power savings are $13.82 \%$ in Sections 1 and 2, but no power savings are recorded in Section 3, as shown in Figure 16(b). The results show that the power savings significantly improve relative to those when the TPC-based distance estimation algorithm is applied.

5.3.2. TPCS/W Technique. When the TPCS/W technique is applied, the generated power savings in the outdoor velodrome are $99.96 \%$ in Section 1 and $99.957 \%$ in Sections 2 and 3, as shown in Figure 17(a). By contrast, in the indoor velodrome, the power savings vary between $99.84 \%$ in Sections 1 and 2 and 99.816\% in Section 3, as shown in Figure 17(b).

The results indicate that the power savings of the mobile node in the outdoor velodrome are higher than those in the indoor velodrome. Such a difference is attributed to the higher propagation path loss in the indoor velodrome. Moreover, the power saved by the mobile node from the use of the TPC-based accelerometer algorithm is higher than that when the TPC-based distance estimation algorithm is used for all the adopted power-saving techniques.

5.4. Comparison between TPC-Based Distance Estimation and TPC-Based Accelerometer Algorithms. To evaluate the 


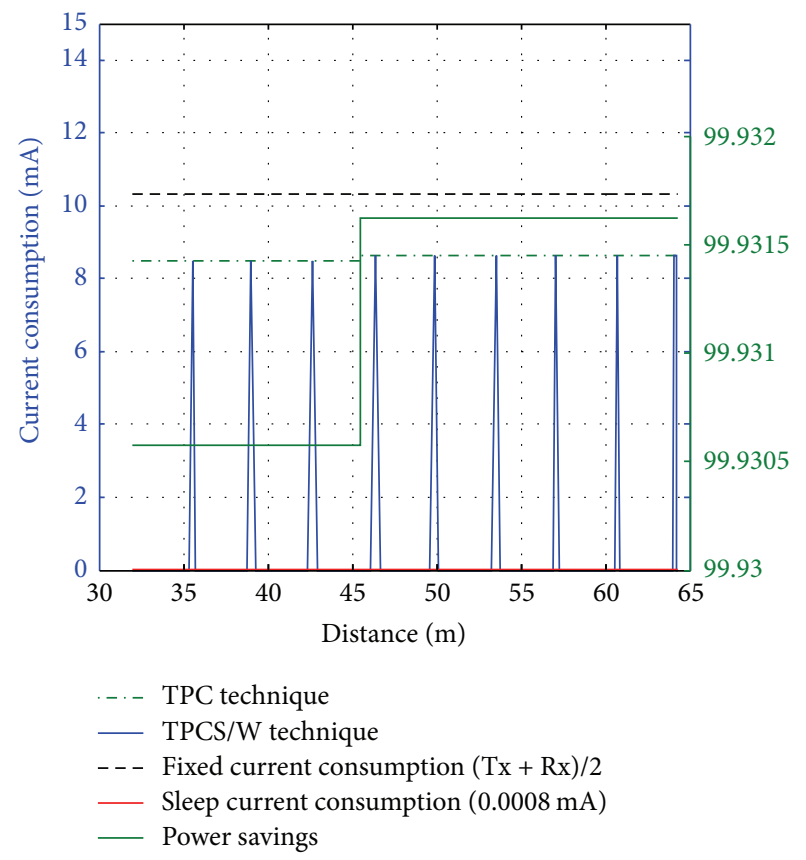

(a)

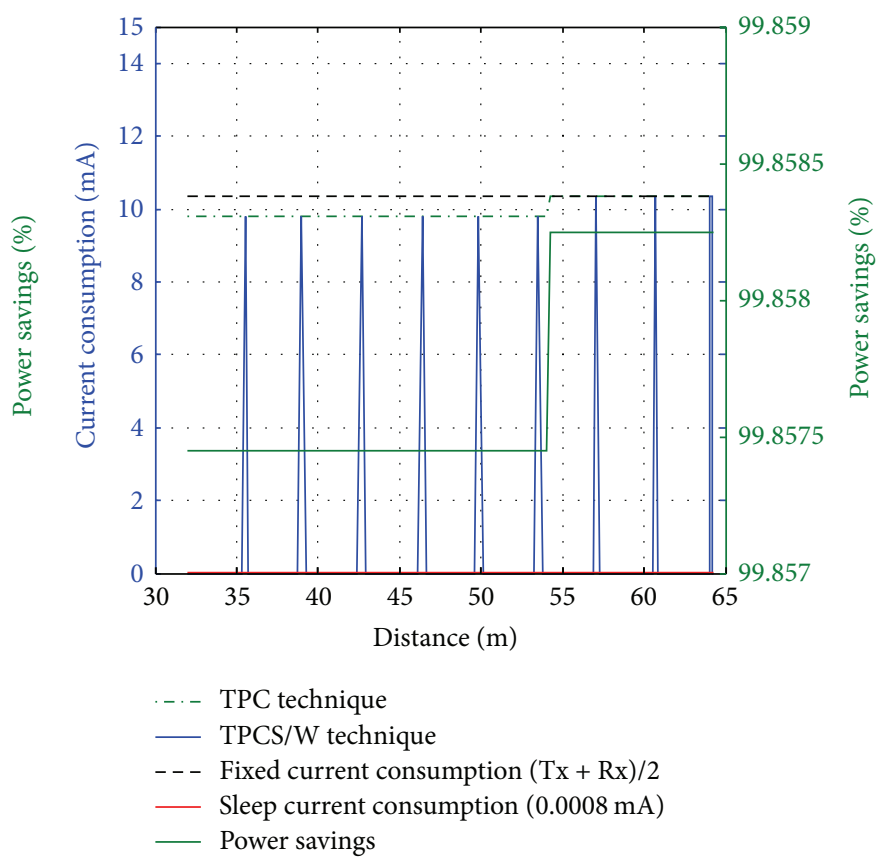

(b)

FIGURE 13: Current consumption and power savings in (a) the outdoor velodrome and (b) the indoor velodrome when applying the TPCW/S algorithm.

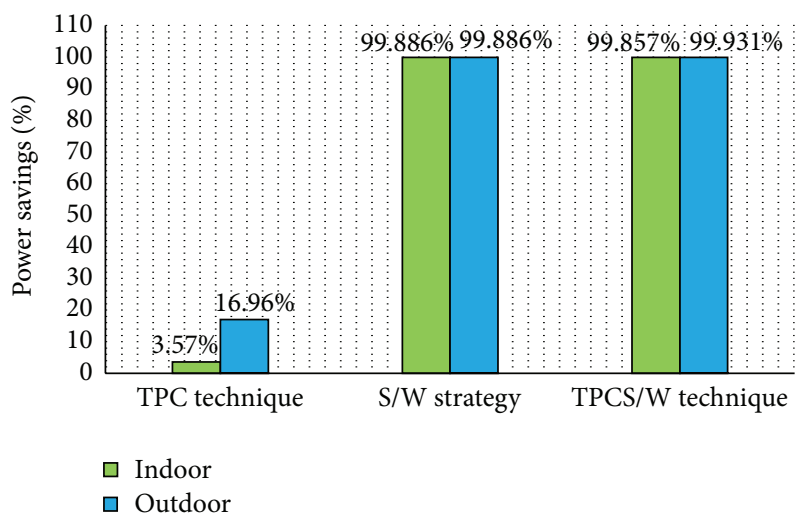

FIGURE 14: Comparison of the adopted power-saving techniques.

ANT power consumption of the mobile node for the two algorithms, the TPC and TPCS/W techniques were compared in terms of power savings, as shown in Figure 18. The figure shows the power savings of the ANT mobile node in the outdoor and indoor velodromes. The comparison results suggest that the average power savings in the outdoor velodrome are greater than those in the indoor velodrome for both algorithms. The average power savings in the outdoor and indoor velodromes for the TPC-based accelerometer algorithm are better than those for the TPCbased distance estimation algorithm. In the outdoor velodrome, the TPC-based accelerometer algorithm produces higher average power savings $55.74 \%$ (obtained from power

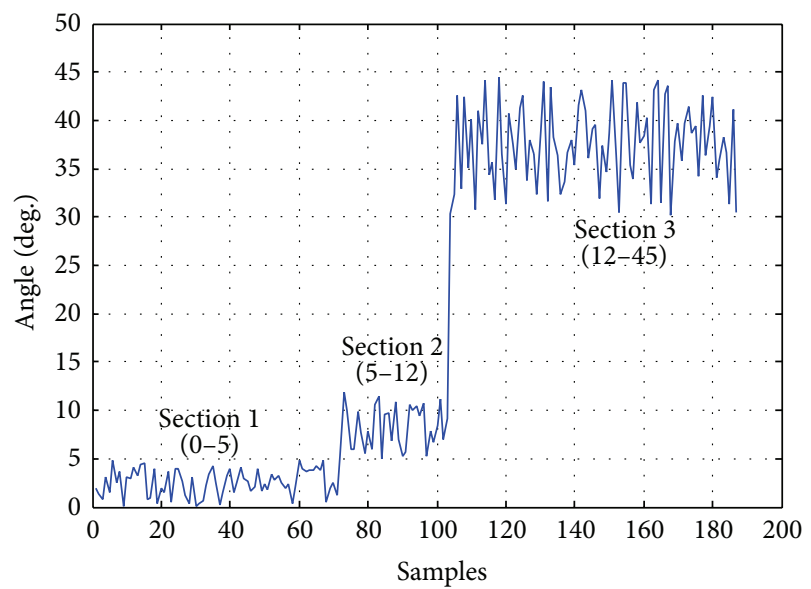

FIGURE 15: Outputs of the accelerometer (inclination angles) according to the three velodrome sections.

savings in [Section $1+$ Section $2+$ Section 3]/3) when the TPC technique is applied. Compared to the TPC-based distance estimation algorithm, the TPC-based accelerometer algorithm generates slightly higher average power savings of $99.95 \%$ (obtained from power savings in [Section $1+$ Section $2+$ Section 3]/3) when the TPCS/W technique is adopted.

The average power savings of the ANT over the entire WSN can also be investigated for both the TPC-based accelerometer and the TPC-based distance estimation algorithms. The average current consumption was previously 


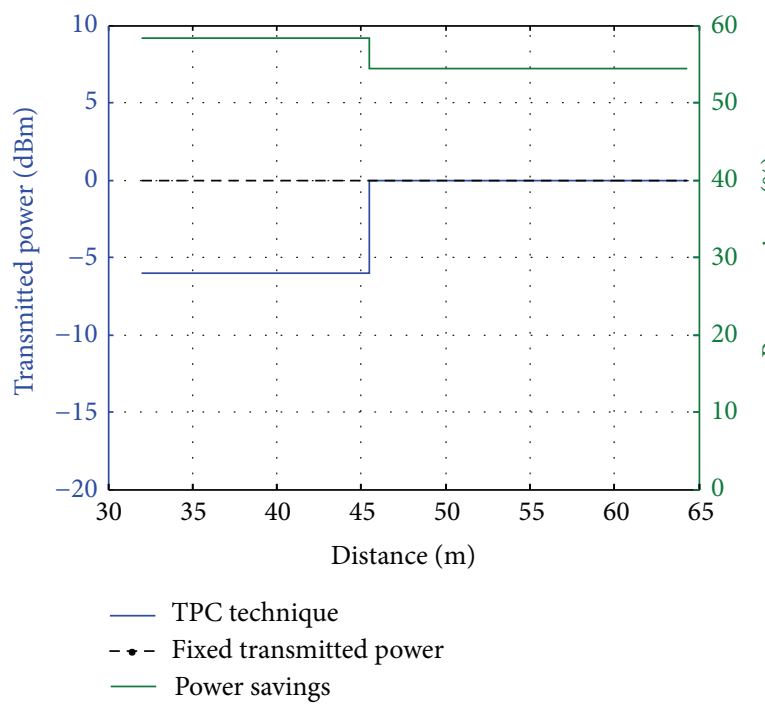

(a)

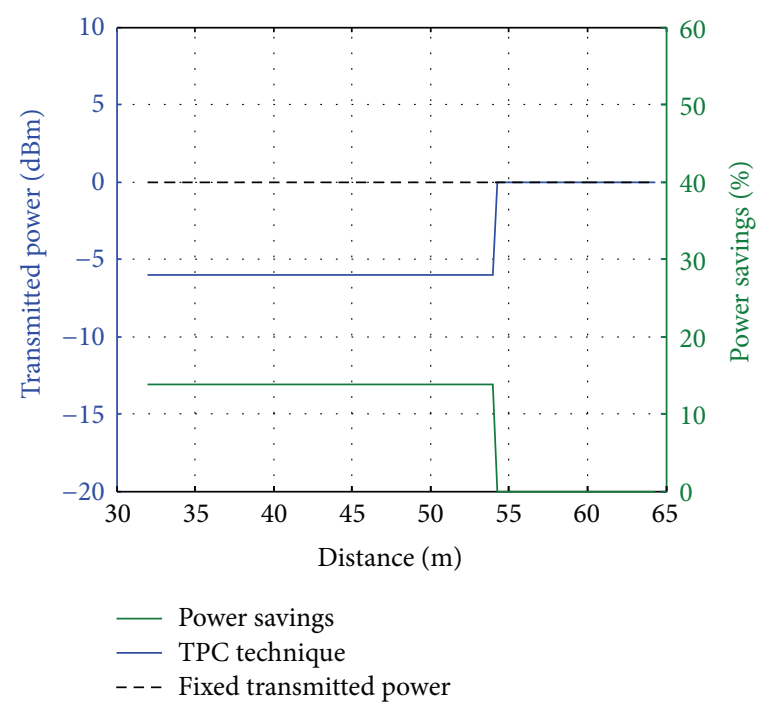

(b)

FIGURE 16: Transmitted power and power savings in (a) the outdoor velodrome and (b) the indoor velodrome obtained by applying the TPC technique.

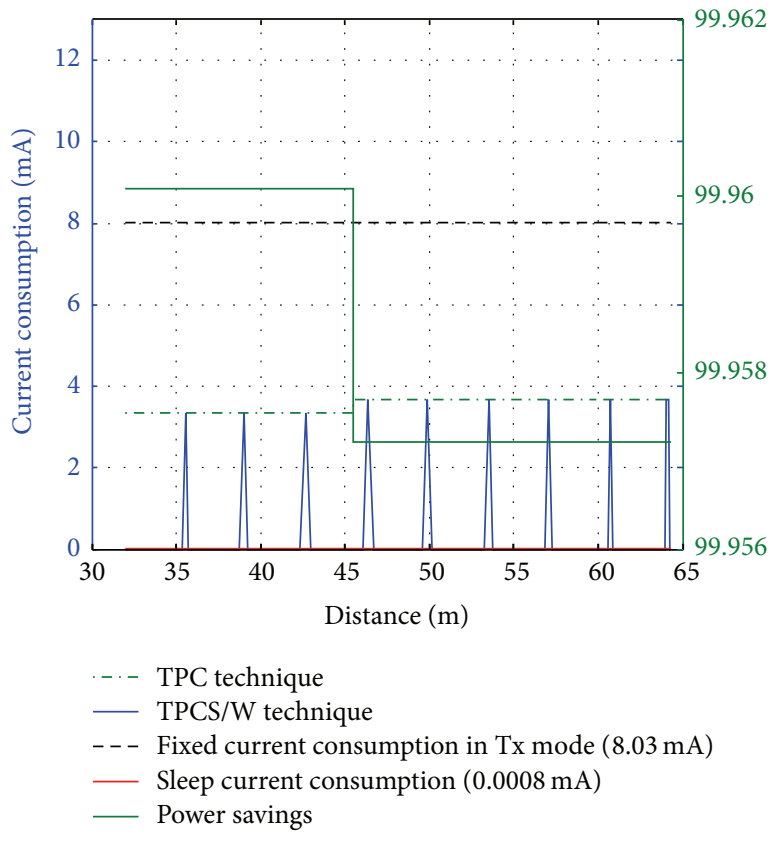

(a)

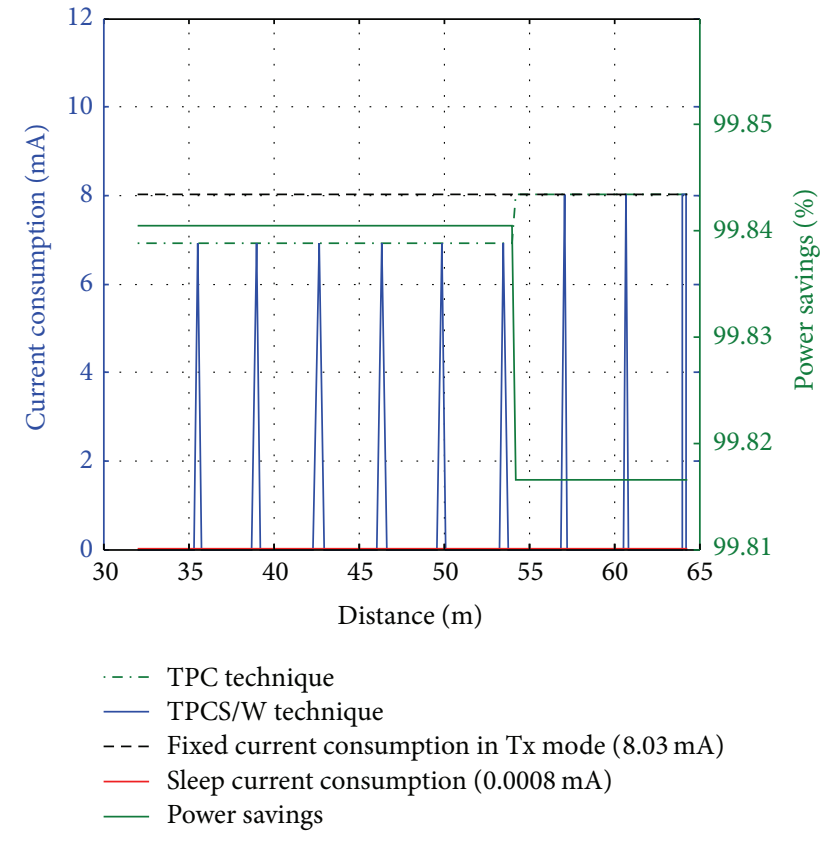

(b)

FIGURE 17: Current consumption and power savings in (a) the outdoor velodrome and (b) the indoor velodrome obtained by applying the TPCW/S algorithm.

obtained, as illustrated in Tables 2 and 3. For the TPC-based accelerometer algorithm, the mobile node only transmits the data collected by the sensors (i.e., Tx mode only), and the master node only receives the data packets (i.e., Rx mode only). Thus, the average ANT current consumption of the mobile and master nodes is 14.733 and $22.751 \mu \mathrm{A}$, respectively. Consequently, the average total ANT current consumption from end to end (i.e., from the mobile node to the master node) is $37.484 \mu \mathrm{A}$.

For the TPC-based distance estimation algorithm, the mobile and master nodes work in both Tx and Rx modes. The average ANT current consumption of the mobile node in the Tx and Rx modes is 14.733 and $12.652 \mu \mathrm{A}$, respectively, whereas that of the master node in the Tx and Rx modes 


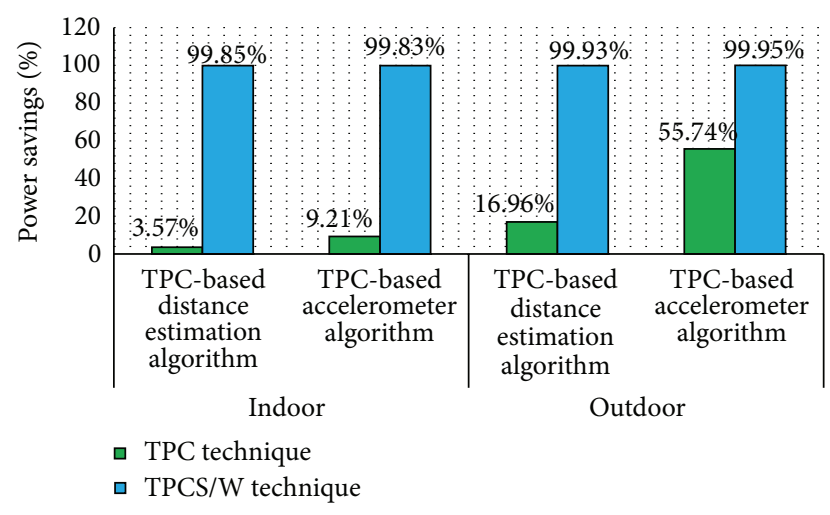

FIGURE 18: Comparison between the TPC-based distance estimation and the TPC-based accelerometer algorithms in both outdoor and indoor velodromes.

is 8.323 and $22.751 \mu \mathrm{A}$, respectively. Therefore, the average total ANT current consumption of the mobile and master nodes is 27.385 and $31.074 \mu \mathrm{A}$, respectively. Accordingly, the average total ANT current consumption from end to end is $58.458 \mu \mathrm{A}$. The average total ANT current consumption values from end to end will be used to compare power savings. The power savings of the TPC-based accelerometer algorithm relative to the TPC-based distance estimation algorithm can be estimated using the following equation:

$$
\begin{aligned}
& \text { Power savings }(\%)=(1 \\
& -\frac{\text { end-to-end average current }}{\text { end-to-end average current }} \text { TPC-based distance }_{\text {TPerometer }} \\
& \times 100 \%
\end{aligned}
$$

Applying (11), power savings of $46 \%, 26 \%$, and $35 \%$ for the ANT mobile node, master node, and the entire ANT in the WSN, respectively, can be achieved using the TPCbased accelerometer algorithm compared to the TPC-based distance estimation algorithm. This result indicates that the TPC-based accelerometer algorithm outperforms the TPCbased distance estimation algorithm. When the TPC-based accelerometer algorithm is applied, the power savings are 99.6\% relative to the fixed transmitted power (i.e., $P_{\mathrm{Tx}}=$ $0 \mathrm{dBm}$ and data rate $=250 \mathrm{kbps}$, where the average current consumption of the mobile and master nodes is $(\mathrm{Tx}+\mathrm{Rx}) / 2=$ $(8.03+12.65)=10.34 \mathrm{~mA})$.

To evaluate the total power consumption of the mobile node, all the active components, such as sensors and microcontroller, must be taken into calculation along with the ANT wireless protocol. The current consumption of all components in the mobile node is shown in Table 7 (obtained through measurement with a FLUKE 15B digital multimeter). The average total current consumption of the mobile node will be used to compare the power savings of the mobile node with other previous approaches based on TPC techniques, namely, $[19,21,23,25-27,66]$, as shown in Figure 19. The results revealed that the TPC-based accelerometer algorithm

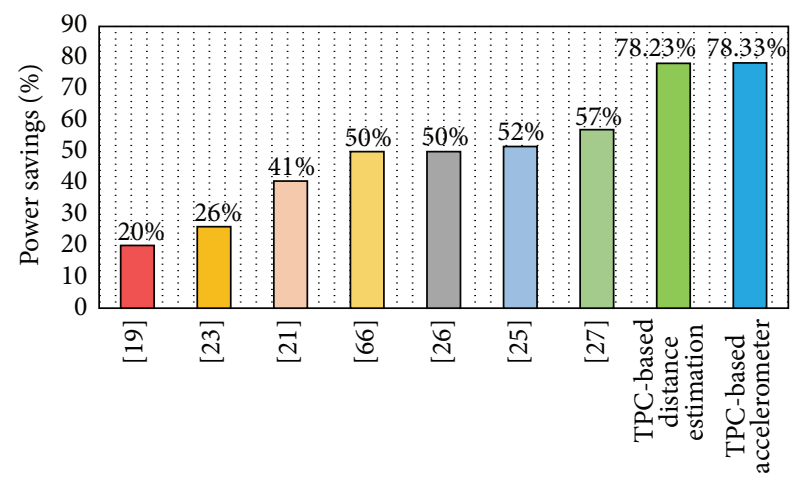

FIGURE 19: Comparison of the TPC-based accelerometer and TPCbased distance estimation algorithms with other TPC techniques employed in previous studies.

and TPC-based distance estimation algorithm outperform the TPC techniques of previous studies, with their power savings of $78 \%$, relative to constant transmitted power.

\section{Conclusion}

In this work, the power consumption of a mobile node moving along a bicycle track was reduced through the use of two proposed algorithms: the TPC-based accelerometer and the TPC-based distance estimation. Both algorithms adopted three power-reduction techniques, namely, a TPC technique, a sleep/wake strategy, and an adjustable data rate, to reduce the power consumption of the ANT mobile node. In addition, the TPC technique was combined with the sleep/wake strategy (TPCS/W) to further reduce the power consumption of the mobile node. The transmitted power level and data rate were arranged in a mapping table to select the optimal transmitted power and data rate that ensure the minimum power consumption and communication connectivity in each section of the outdoor and indoor velodromes. The results indicated not only that the control of the transmitted power level can reduce the power consumption but also that the data rate has a significant effect on power consumption. The combination of the three techniques significantly reduced power consumption by more than $99 \%$. The TPC-based accelerometer algorithm outperformed the TPC-based distance estimation algorithm by $35 \%$ for the entire ANTs in the WSN in terms of power savings. Both TPC-based accelerometer and TPC-based distance estimation algorithms outperformed the previous research works in terms of power savings, where these algorithms achieved $78 \%$ power saving relative to conventional operation (i.e., without power reduction algorithm). Relationships between the ANT data rate and the average current consumption of the mobile and master nodes in the Tx and Rx modes were established. These relationships facilitated the average current consumption calculation. Our future work will focus on implementing the TPC-based accelerometer algorithm on the testbed. 


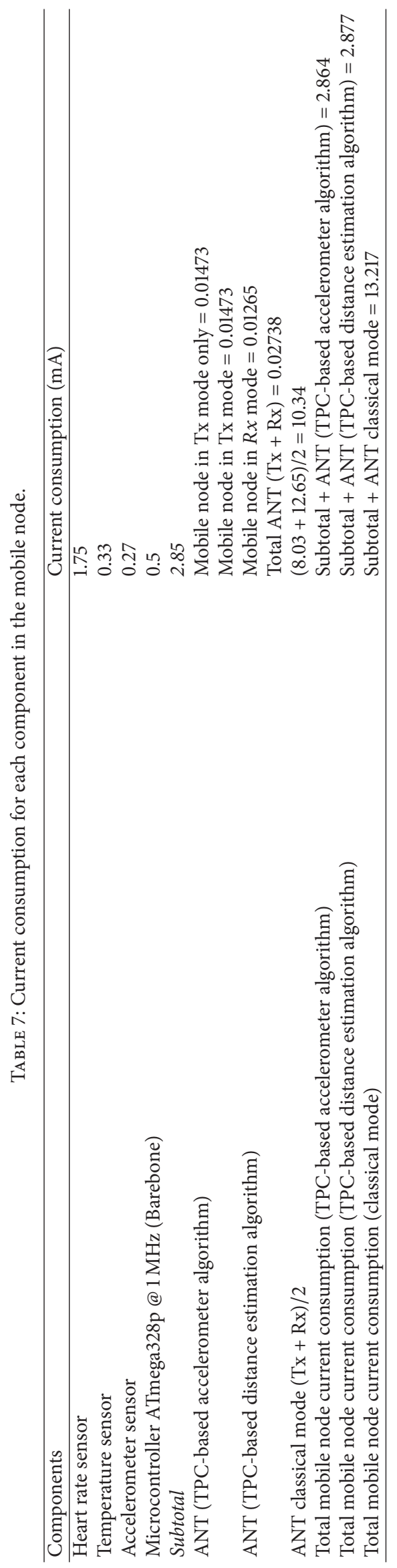




\section{Competing Interests}

The authors declare that there is no conflict of interests regarding the publication of this paper.

\section{Acknowledgments}

This work was supported by Malaysia’s Ministry of Education, under Sports Engineering Grant with ref. no. PKT1/2013, and Universiti Kebangsaan Malaysia with Grant ref. no. INOVASI-2014-015.

\section{References}

[1] N. S. Kulkarni, R. Rakesh, S. Bhargava, S. S. Bundela, and R. Hegde, "Zigbee based low power Wireless Sensor Network motes," in Proceedings of the International Conference on Next Generation Networks, pp. 1-6, Mumbai, India, September 2010.

[2] P. T. V. Bhuvaneswari, V. Vaidehi, and M. Agnes Saranya, "Optimal transmission power selection under energy constraints for sensor network localization," in Proceedings of the 11th International Conference on Control, Automation, Robotics \& Vision (ICARCV'10), pp. 443-447, Singapore, December 2010.

[3] R. Sudarmani and K. Kumar, "Analysis of energy consumption in heterogeneous sensor networks with mobile sink," in Proceedings of the 10th IEEE Conference on TENCON, pp. 455-459, 2011.

[4] M. Al Ameen, S. M. R. Islam, and K. Kwak, "Energy saving mechanisms for MAC protocols in wireless sensor networks," International Journal of Distributed Sensor Networks, vol. 2010, Article ID 163413, 16 pages, 2010.

[5] H. Asgarizadeh and J. Abouei, "An energy-efficient SD-based LZW algorithm in dynamic wireless sensor networks," in Proceedings of the 21st Iranian Conference on Electrical Engineering (ICEE '13), pp. 1-6, Mashhad, Iran, May 2013.

[6] C. Buratti, A. Conti, D. Dardari, and R. Verdone, "An overview on wireless sensor networks technology and evolution," Sensors, vol. 9, no. 9, pp. 6869-6896, 2009.

[7] G. Anastasi, M. Conti, M. Di Francesco, and A. Passarella, "Energy conservation in wireless sensor networks: a survey," Ad Hoc Networks, vol. 7, no. 3, pp. 537-568, 2009.

[8] C. Zhen, W. Liu, Y. Liu, and A. Yan, "Energy-efficient sleep/wake scheduling for acoustic localization wireless sensor network node," International Journal of Distributed Sensor Networks, vol. 2014, Article ID 970524, 14 pages, 2014.

[9] J. Azevedo, F. Santos, M. Rodrigues, and L. Aguiar, "Sleeping ZigBee networks at the application layer," IET Wireless Sensor Systems, vol. 4, no. 1, pp. 35-41, 2014.

[10] Y. Wang, H. Chen, X. Wu, and L. Shu, "An energy-efficient SDN based sleep scheduling algorithm for WSNs," Journal of Network and Computer Applications, vol. 59, pp. 39-45, 2016.

[11] C.-M. Chao and T.-Y. Hsiao, "Design of structure-free and energy-balanced data aggregation in wireless sensor networks," Journal of Network and Computer Applications, vol. 37, no. 1, pp. 229-239, 2014.

[12] M. N. Rahman and M. A. Matin, "Efficient algorithm for prolonging network lifetime of wireless sensor networks," Tsinghua Science \& Technology, vol. 16, no. 6, pp. 561-568, 2011.

[13] J.-S. Lee and W.-L. Cheng, "Fuzzy-logic-based clustering approach for wireless sensor networks using energy predication," IEEE Sensors Journal, vol. 12, no. 9, pp. 2891-2897, 2012.
[14] L. Mesin, S. Aram, and E. Pasero, "A neural data-driven algorithm for smart sampling in wireless sensor networks," EURASIP Journal on Wireless Communications and Networking, vol. 2014, article 23, pp. 1-8, 2014.

[15] S. A. Imtiaz, A. J. Casson, and E. Rodriguez-Villegas, "Compression in wearable sensor nodes: impacts of node topology," IEEE Transactions on Biomedical Engineering, vol. 61, no. 4, pp. 10801090, 2014.

[16] S. Rizvi, H. K. Qureshi, S. Ali Khayam, V. Rakocevic, and M. Rajarajan, "A1: an energy efficient topology control algorithm for connected area coverage in wireless sensor networks," Journal of Network and Computer Applications, vol. 35, no. 2, pp. 597-605, 2012.

[17] “2.4 GHZ + NRF24L01 Wireless Module," 2015, https://www .fasttech.com/product/1215200-24ghz-nrf24l01-wireless-module.

[18] D. Gao, G. Wu, Y. Liu, and F. Zhang, "Bounded end-to-end delay with Transmission Power Control techniques for rechargeable wireless sensor networks," International Journal of Electronics and Communications, vol. 68, no. 5, pp. 395-405, 2014.

[19] H. Cotuk, K. Bicakci, B. Tavli, and E. Uzun, "The impact of transmission power control strategies on lifetime of wireless sensor networks," IEEE Transactions on Computers, vol. 63, no. 11, pp. 2866-2879, 2014.

[20] D.-Y. Gao, L.-J. Zhang, and H.-C. Wang, "Energy saving with node sleep and power control mechanisms for wireless sensor networks," The Journal of China Universities of Posts and Telecommunications, vol. 18, no. 1, pp. 49-59, 2011.

[21] S. Ramakrishnan and B. T. Krishna, "Closed loop fuzzy logic based transmission power control for energy efficiency in wireless sensor networks," in Proceedings of the IEEE International Conference on Computer Communication and Systems (ICCCS '14), pp. 195-200, Chennai, India, February 2014.

[22] J. Lee and K. Chung, "An efficient transmission power control scheme for temperature variation in wireless sensor networks," Sensors, vol. 11, no. 3, pp. 3078-3093, 2011.

[23] A. Castagnetti, A. Pegatoquet, T. N. Le, and M. Auguin, "A joint duty-cycle and transmission power management for energy harvesting WSN," IEEE Transactions on Industrial Informatics, vol. 10, no. 2, pp. 928-936, 2014.

[24] G. Dai, J. Qiu, P. Liu, B. Lin, and S. Zhang, "Remaining energylevel-based transmission power control for energy-harvesting WSNs," International Journal of Distributed Sensor Networks, vol. 2012, Article ID 934240, 12 pages, 2012.

[25] S. Kim, S. Kim, and D.-S. Eom, "RSSI/LQI-based transmission power control for body area networks in healthcare environment," IEEE Journal of Biomedical and Health Informatics, vol. 17, no. 3, pp. 561-571, 2013.

[26] W. Ikram, S. Petersen, P. Orten, and N. F. Thornhill, "Adaptive multi-channel transmission power control for industrial wireless instrumentation," IEEE Transactions on Industrial Informatics, vol. 10, no. 2, pp. 978-990, 2014.

[27] L. H. A. Correia, D. F. Macedo, A. L. dos Santos, A. A. F. Loureiro, and J. M. S. Nogueira, "Transmission power control techniques for wireless sensor networks," Computer Networks, vol. 51, no. 17, pp. 4765-4779, 2007.

[28] K. Benkič, M. Malajner, P. Planinšič, and Ž. Čučej, "Using RSSI value for distance estimation in Wireless sensor networks based on ZigBee," in Proceedings of the 15th International Conference on Systems, Signals and Image Processing (IWSSIP '08), pp. 303306, Bratislava, Slovakia, June 2008.

[29] O. Musikanon and W. Chongburee, "ZigBee propagations and performance analysis in last mile network," International 
Journal of Innovation and Technology Management, vol. 3, pp. 353-357, 2012.

[30] M. Malajner, K. Benkič, P. Planinšič, and Ž. Čučej, "The accuracy of propagation models for distance measurement between WSN nodes," in Proceedings of the 16th International Conference on Systems, Signals and Image Processing (IWSSIP '09), pp. 1-4, IEEE, Chalkida, Greece, June 2009.

[31] Y. S. Cho, J. Kim, W. Y. Yang, and C. G. Kang, MIMO-OFDM Wireless Communications with MATLAB, John Wiley \& Sons, New York, NY, USA, 2010.

[32] S. Kim and D.-S. Eom, "Distributed transmission power control for network programming in wireless sensor networks," Wireless Personal Communications, vol. 72, no. 2, pp. 1533-1548, 2013.

[33] P.-J. Chuang and Y.-J. Jiang, "Effective neural network-based node localisation scheme for wireless sensor networks," IET Wireless Sensor Systems, vol. 4, no. 2, pp. 97-103, 2014.

[34] P. K. Sahu, E. H.-K. Wu, and J. Sahoo, "DuRT: dual RSSI trend based localization for wireless sensor networks," IEEE Sensors Journal, vol. 13, no. 8, pp. 3115-3123, 2013.

[35] E. T. Yazdi, A. Willig, and K. Pawlikowski, "Frequency adaptation for interference mitigation in IEEE 802.15.4-based mobile body sensor networks," Computer Communications, vol. 53, pp. 102-119, 2014.

[36] P. Tarrío, A. M. Bernardos, and J. R. Casar, "An energy-efficient strategy for accurate distance estimation in wireless sensor networks," Sensors, vol. 12, no. 11, pp. 15438-15466, 2012.

[37] R.-B. Zhang, J.-G. Guo, F.-H. Chu, and Y.-C. Zhang, "Environmental-adaptive indoor radio path loss model for wireless sensor networks localization," International Journal of Electronics and Communications, vol. 65, no. 12, pp. 1023-1031, 2011.

[38] P. Tarrío, A. M. Bernardos, and J. R. Casar, "Weighted least squares techniques for improved received signal strength based localization," Sensors, vol. 11, no. 9, pp. 8569-8592, 2011.

[39] Y. Sadi, S. C. Ergen, and P. Park, "Minimum energy data transmission for wireless networked control systems," IEEE Transactions on Wireless Communications, vol. 13, no. 4, pp. 2163-2175, 2014.

[40] S. K. Gharghan, R. Nordin, and M. Ismail, "An ultra-low power wireless sensor network for bicycle torque performance measurements," Sensors, vol. 15, no. 5, pp. 11741-11768, 2015.

[41] G. Selimis, L. Huang, F. Massé et al., "A lightweight security scheme for wireless body area networks: design, energy evaluation and proposed microprocessor design," Journal of Medical Systems, vol. 35, no. 5, pp. 1289-1298, 2011.

[42] NORDIC Semiconductor, nRF24L01 Ultra Low Power $2.4 \mathrm{GHz}$ RF Transceiver, http://www.nordicsemi.com/eng/Products/ 2.4GHz-RF/nRF24L01.

[43] L.-Z. Zhao, X.-B. Wen, and D. Li, "Amorphous localization algorithm based on BP artificial neural network," International Journal of Distributed Sensor Networks, vol. 2015, Article ID 657241, 9 pages, 2015.

[44] M. Gholami, N. Cai, and R. W. Brennan, "An artificial neural network approach to the problem of wireless sensors network localization," Robotics and Computer-Integrated Manufacturing, vol. 29, no. 1, pp. 96-109, 2013.

[45] T. Rault, A. Bouabdallah, and Y. Challal, "Energy efficiency in wireless sensor networks: a top-down survey," Computer Networks, vol. 67, pp. 104-122, 2014.

[46] A. Santos-Lozano, P. J. Marín, G. Torres-Luque, J. R. Ruiz, A. Lucía, and N. Garatachea, "Technical variability of the GT3X accelerometer," Medical Engineering \& Physics, vol. 34, no. 6, pp. 787-790, 2012.

[47] A. Olivares, G. Olivares, F. Mula, J. M. Górriz, and J. Ramírez, "Wagyromag: wireless sensor network for monitoring and processing human body movement in healthcare applications," Journal of Systems Architecture, vol. 57, no. 10, pp. 905-915, 2011.

[48] R. Dorado-Vicente, P. Romero-Carrillo, R. Lopez-Garcia, and F. A. Diaz-Garrido, "Comparing planar pocketing tool paths via acceleration measurement," Procedia Engineering, vol. 63, pp. 270-277, 2013.

[49] L. Atallah, A. Wiik, G. G. Jones et al., "Validation of an ear-worn sensor for gait monitoring using a force-plate instrumented treadmill," Gait \& Posture, vol. 35, no. 4, pp. 674-676, 2012.

[50] D. Kukolj and E. Levi, "Identification of complex systems based on neural and takagi-sugeno fuzzy model," IEEE Transactions on Systems, Man, and Cybernetics, Part B: Cybernetics, vol. 34, no. 1, pp. 272-282, 2004.

[51] M. Abdelhadi, M. Anan, and M. Ayyash, "Efficient artificial intelligent-based localization algorithm for wireless sensor networks," Cyber Journals: Multidisciplinary Journals in Science and Technology, Journal of Selected Areas in Telecommunications (JSAT), vol. 3, pp. 10-18, 2013.

[52] C. Nerguizian and V. Nerguizian, "Indoor fingerprinting geolocation using wavelet-based features extracted from the channel impulse response in conjunction with an artificial neural network," in Proceedings of the IEEE International Symposium on Industrial Electronics (ISIE '07), pp. 2028-2032, IEEE, Vigo, Spain, June 2007.

[53] A. Azenha, L. Peneda, and A. Carvalho, "A neural network approach for Radio Frequency based indoors localization," in Proceedings of the 38th Annual Conference on IEEE Industrial Electronics Society (IECON '12), pp. 5990-5995, IEEE, Montreal, Canada, October 2012.

[54] M. S. Rahman, Y. Park, and K.-D. Kim, "RSS-based indoor localization algorithm for wireless sensor network using generalized regression neural network," Arabian Journal for Science and Engineering, vol. 37, no. 4, pp. 1043-1053, 2012.

[55] A. Payal, C. S. Rai, and B. V. R. Reddy, "Artificial neural networks for developing localization framework in wireless sensor networks," in Proceedings of the International Conference on Data Mining and Intelligent Computing (ICDMIC '14), pp. 16, IEEE, New Delhi, India, September 2014.

[56] S. M. Nekooei and M. T. Manzuri-Shalmani, "Location finding in wireless sensor network based on soft computing methods," in Proceedings of the International Conference on Control, Automation and Systems Engineering (CASE '11), pp. 1-5, Singapore, July 2011.

[57] W. Gao, G. Kamath, K. Veeramachaneni, and L. Osadciw, "A particle swarm optimization based multilateration algorithm for UWB sensor network," in Proceedings of the Canadian Conference on Electrical and Computer Engineering (CCECE '09), pp. 950-953, IEEE, St. John's, Canada, May 2009.

[58] S. Yun, J. Lee, W. Chung, E. Kim, and S. Kim, "A soft computing approach to localization in wireless sensor networks," Expert Systems with Applications, vol. 36, no. 4, pp. 7552-7561, 2009.

[59] A. Payal, C. S. Rai, and B. V. R. Reddy, "Comparative analysis of Bayesian regularization and Levenberg-Marquardt training algorithm for localization in wireless sensor network," in Proceedings of the 15th International Conference on Advanced Communication Technology (ICACT '13), pp. 191-194, PyeongChang, South Korea, January 2013. 
[60] R. Parthiban and A. Menon, "A fuzzy logic algorithm for minimizing error (FLAME) in wireless sensor networks," in Proceedings of the IEEE/ASME International Conference on Advanced Intelligent Mechatronics (AIM '09), pp. 1435-1440, Singapore, July 2009.

[61] L. Gogolak, S. Pletl, and D. Kukolj, "Indoor fingerprint localization in WSN environment based on neural network," in Proceedings of the 9th International Symposium on Intelligent Systems and Informatics (SISY '11), pp. 293-296, IEEE, Subotica, Serbia, September 2011.

[62] A. Payal, C. S. Rai, and B. V. R. Reddy, "Analysis of some feedforward artificial neural network training algorithms for developing localization framework in wireless sensor networks," Wireless Personal Communications, vol. 82, no. 4, pp. 2519-2536, 2015.

[63] T. Zhang, J. He, and Y. Zhang, "Secure sensor localization in wireless sensor networks based on neural network," International Journal of Computational Intelligence Systems, vol. 5, no. 5, pp. 914-923, 2012.

[64] G. Yang, Z. Yi, N. Tianquan, Y. Keke, and X. Tongtong, "An improved genetic algorithm for wireless sensor networks localization," in Proceedings of the IEEE 5th International Conference on Bio-Inspired Computing: Theories and Applications (BIC-TA '10), pp. 439-443, IEEE, Changsha, China, September 2010.

[65] A. O. de Sá, N. Nedjah, and L. de Macedo Mourelle, "Distributed efficient localization in swarm robotic systems using swarm intelligence algorithms," Neurocomputing, vol. 172, pp. 322-336, 2016.

[66] R. Yan, H. Sun, and Y. Qian, "Energy-aware sensor node design with its application in wireless sensor networks," IEEE Transactions on Instrumentation and Measurement, vol. 62, no. 5, pp. 1183-1191, 2013. 


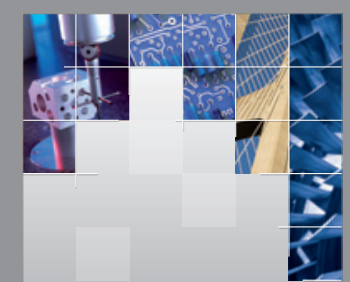

\section{Enfincering}
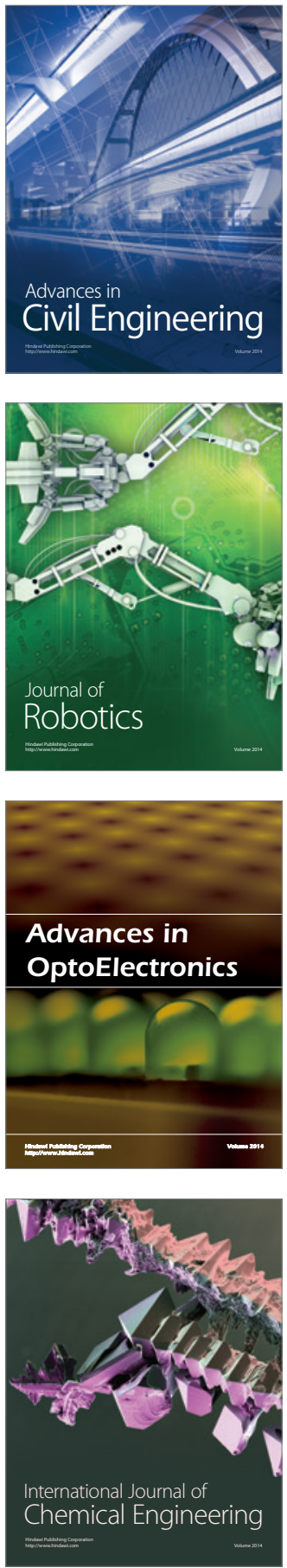

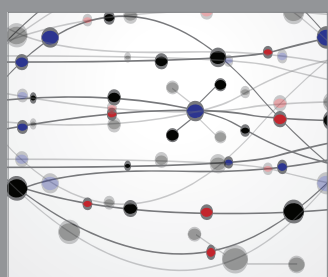

The Scientific World Journal

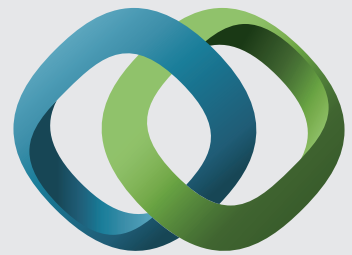

\section{Hindawi}

Submit your manuscripts at

http://www.hindawi.com
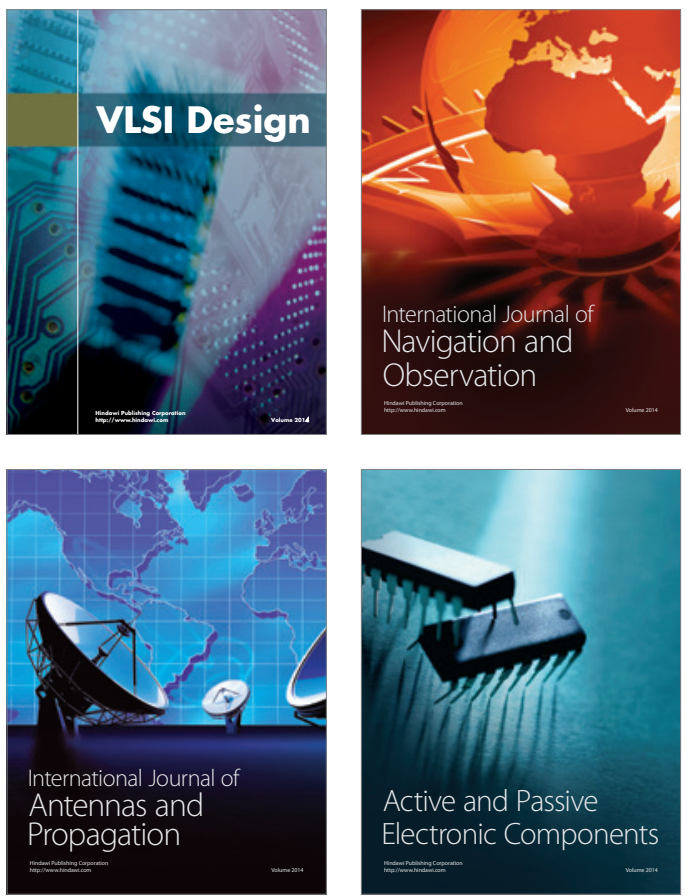
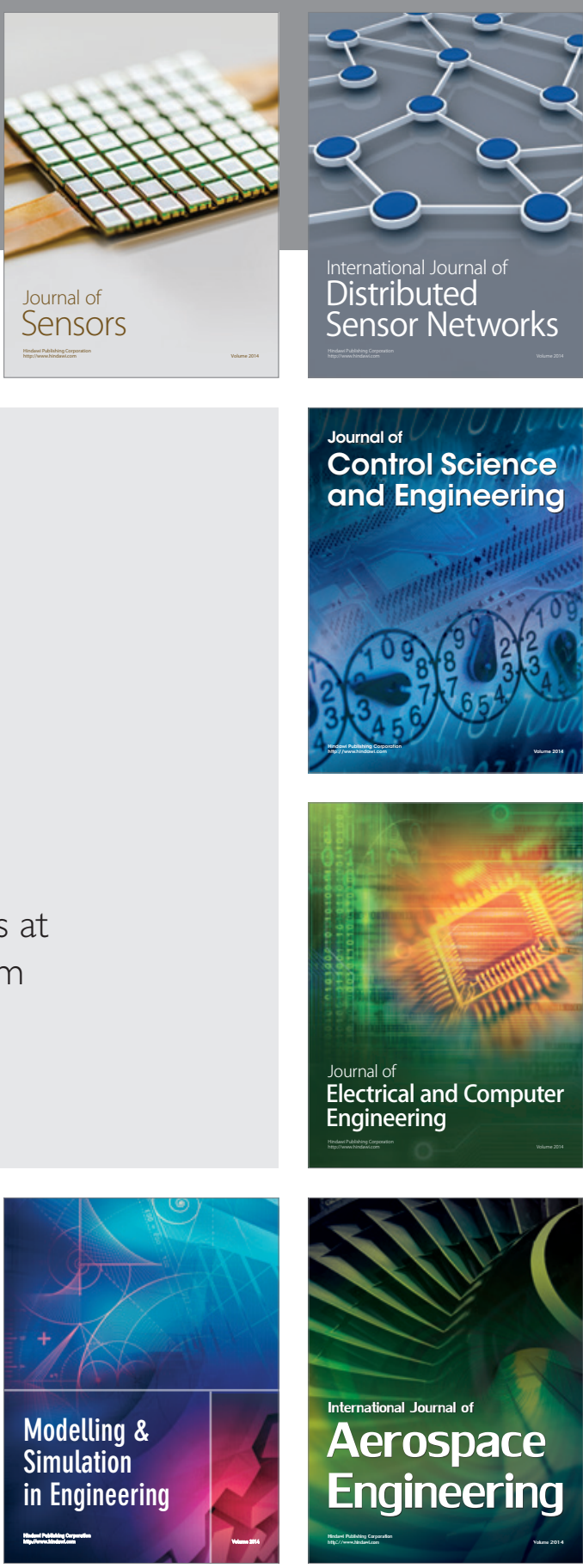

International Journal of

Distributed

Sensor Networks

Journal of

Control Science

and Engineering
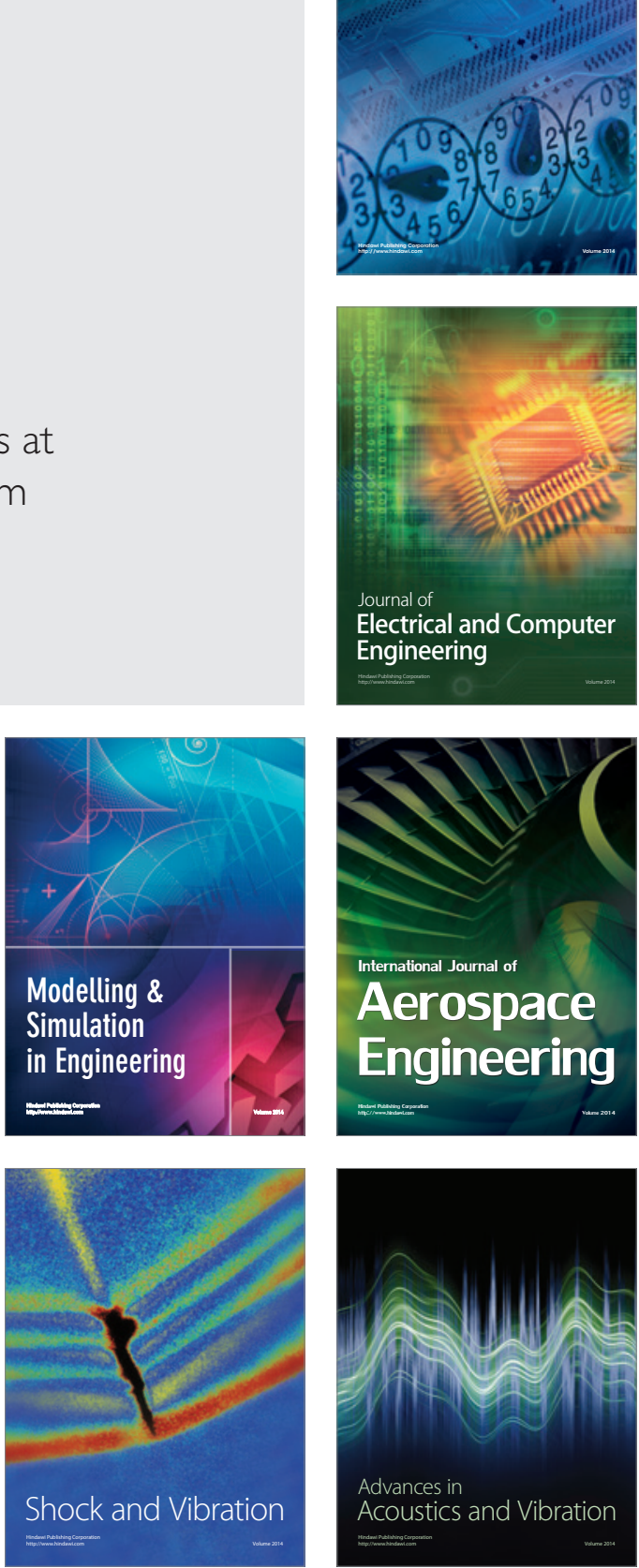